\title{
Effects of Chemical Reactions on Unsteady Free Convective and Mass Transfer Flow from a Vertical Cone with Heat Generation/Absorption in the Presence of VWT/VWC
}

\author{
Bapuji Pullepu, ${ }^{1}$ P. Sambath, ${ }^{1}$ and K. K. Viswanathan ${ }^{2}$ \\ ${ }^{1}$ Department of Mathematics, SRM University, Kattankulathur, Tamil Nadu 603203, India \\ ${ }^{2}$ UTM Centre for Industrial and Applied Mathematics and Department of Mathematical Sciences, Faculty of Science, \\ Universiti Teknologi Malaysia, 81310 Johor Bahru, Johor, Malaysia
}

Correspondence should be addressed to K. K. Viswanathan; visu20@yahoo.com

Received 19 April 2014; Revised 19 June 2014; Accepted 19 June 2014; Published 24 July 2014

Academic Editor: Ebrahim Momoniat

Copyright (C) 2014 Bapuji Pullepu et al. This is an open access article distributed under the Creative Commons Attribution License, which permits unrestricted use, distribution, and reproduction in any medium, provided the original work is properly cited.

\begin{abstract}
A mathematical model for the effects of chemical reaction and heat generation/absorption on unsteady laminar free convective flow with heat and mass transfer over an incompressible viscous fluid past a vertical permeable cone with nonuniform surface temperature $T_{w}^{\prime}(x)=T_{\infty}^{\prime}+a x^{n}$ and concentration $C_{w}^{\prime}(x)=C_{\infty}^{\prime}+b x^{m}$ is considered here. The dimensionless governing boundary layer equations of the flow that are transient, coupled, and nonlinear partial differential equations are solved by an efficient, accurate, and unconditionally stable finite difference scheme of Crank-Nicholson type. The velocity, temperature, and concentration profiles have been studied for various parameters, namely, chemical reaction parameter $\lambda$, the heat generation and absorption parameter $\Delta$, Schmidt number Sc, Prandtl number Pr, buoyancy ratio parameter $N$, surface temperature power law exponent $n$, and surface concentration power law exponent $m$. The local as well as average skin friction, Nusselt number, and Sherwood number are discussed and analyzed graphically. The present results are compared with available results in open literature and are found to be in excellent agreement.
\end{abstract}

\section{Introduction}

The problem of two-dimensional axisymmetric free convective flow past a vertical cone with different boundary conditions has attracted the attention of many researchers in recent years. When a heated surface is in contact with the fluid, the result of temperature difference causes buoyancy force, which induces the natural convection. Free convection flows under the influence of gravitational force have been studied in detail because they occur frequently in nature. Simultaneous heat and mass transfer in natural convection flows on a vertical cone has a wide range of applications in the field of science and technology. Also it plays an important role in manufacturing industries for the design of reliable equipment for nuclear power plants, gas turbines, and various propulsion devices for aircraft, missiles, satellites, and space vehicles. The flow of a fluid is caused not only by the temperature differences, but also by concentration differences. These concentration differences affect the flow and temperature near the surface of a body embedded in a fluid. In engineering applications, the concentration differences are created either by injecting the foreign gases or by coating the surface with evaporating material which evaporates due to the heat of the surface. The presence of foreign masses in air and water like hydrogen $\mathrm{H}_{2}$ and water $\mathrm{H}_{2} \mathrm{O}$ causes some kind of chemical reaction. Heat is generated due to this chemical reaction. A common example of heat and mass transfer is the evaporation of lake water into the wind flowing over it. In some cases, mass transfer is predominant and heat transfer may be negligible; in other cases, both remain equally predominant. Mass transfer proceeds as long as there is a difference in concentrations of some chemical 


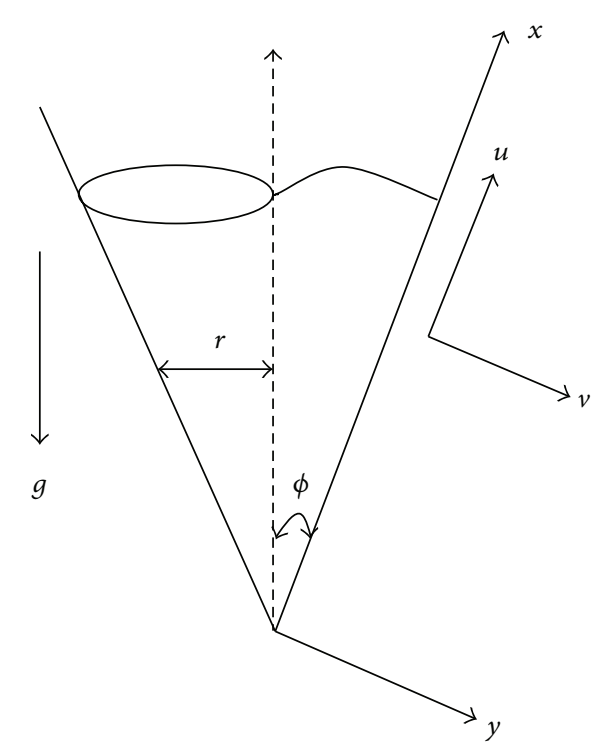

FIgUre 1: Physical model and coordinate system.

species in the mixture. Hence, the concentration gradient acts as a driving potential in mass transfer, just as the temperature gradient does in heat transfer.

Since 1953 several authors have developed similarity/nonsimilarity solutions for two-dimensional axisymmetrical problems for natural convection laminar flow over vertical cone in steady state (see [1-4]). Kafoussias [5] analyzed the effects of mass transfer on a free convective flow past a vertical cone surface embedded in an infinite, incompressible, and viscous fluid. Yih [6,7] studied in saturated porous media the combined heat and mass transfer effects over a full cone with uniform wall temperature/concentration or heat/mass flux and for truncated cone with nonuniform wall temperature/variable wall concentration or variable heat/mass flux using the Keller box implicit difference method. Chamkha [8] considered the problem of steady state laminar heat and mass transfer by natural convection boundary layer flow around a permeable truncated cone in the presence of magnetic field and thermal radiation effects; nonsimilar solutions were obtained and solved numerically by an implicit finite difference methodology. Later Chamkha and Quadri [9] solved the problem of combined heat and mass transfer by hydromagnetic natural convection over a cone embedded in a non-Darcian porous medium with heat generation/absorption effects; a nonsimilar form of the solution was solved numerically by an implicit, iterative, and finite difference method. Afify [10] studied the effects of radiation and chemical reaction on a steady free convective flow and mass transfer of an optically dense viscous, incompressible, and electrically conducting fluid past a vertical isothermal cone in the presence of a magnetic field; the resulting similarity equations were solved numerically using a fourth-order Runge-Kutta scheme with the shooting technique. Chamkha and Al-Mudhaf [11] focused on the study of unsteady heat and mass transfer by mixed convection flow over a vertical permeable cone rotating in an ambient fluid with a time-dependent angular velocity in the presence of a magnetic field and heat generation or absorption effects with the cone surface which is maintained at variable temperature and concentration and obtained numerical solutions by solving the governing partial differential equations using an implicit, iterative finite difference scheme. Chamkha et al. [12] studied the effects of coupled heat and mass transfer by boundary layer free convection over a vertical flat plate embedded in a fluidsaturated porous medium in the presence of thermophoretic particle deposition and heat generation or absorption effects; the governing partial differential equations are transformed into ordinary differential equations by using special transformations and the resulting similarity equations are solved numerically by an efficient implicit tridiagonal finite difference method. EL-Kabeir et al. [13] used perturbation method to study the effect of heat and mass transfer on free convection flow with a uniform suction and injection over a cone in a micropolar fluid. EL-Kabeir and Abdou [14] studied the effects of chemical reaction and heat and mass transfer on MHD flow over a vertical isothermal cone surface in micropolar fluids with heat generation/absorption effects and obtained numerical solutions by using the fourthorder Runge-Kutta method with shooting technique. Also ElKabeir et al. [15] discussed the linear transformation group approach to simulate the problem of heat and mass transfer in steady, two-dimensional, laminar, and boundary layer flow of a viscous, incompressible, and electrically conducting fluid over a vertical permeable cone surface saturated porous medium in the presence of a uniform transverse magnetic field and thermal radiation effects. Cheng [16] presented a boundary layer analysis about the natural convection heat and mass transfer near a vertical cone with variable wall temperature and concentration in a porous medium saturated with non-Newtonian power law fluids; coordinate transform is used to obtain the nonsimilar governing equations, and the transformed boundary layer equations are solved by the cubic spline collocation method. Cheng $[17,18]$ analyzed the Soret and Dufour effects on the boundary layer flow due to natural convection heat and mass transfer over a downward-pointing vertical cone and truncated cone in a porous medium saturated with Newtonian fluids with constant wall temperature and concentration, similarity analysis is performed, and similarity equations are solved by cubic spline collocation method. Murti et al. [19] discussed the radiation and chemical reaction effects on heat and mass transfer in non-Darcy nonNewtonian fluid over a vertical surface; the governing boundary layer equations and boundary conditions are simplified by using similarity transformations and are solved numerically by means of fourth-order Runge-Kutta method coupled with double-shooting technique. Kishore et al. [20] studied viscoelastic buoyancy driven MHD free convective heat and mass transfer past a vertical cone with thermal radiation and viscous dissipation and obtained numerical solutions for the governing equations using Crank-Nicholson method. Mahdy [21] focused on the study of combined heat and mass transfer on double-diffusive convection near a vertical truncated cone in a fluid-saturated porous medium in the presence of a firstorder chemical reaction and heat generation or absorption with variable viscosity. Viscosity of the fluid is assumed 

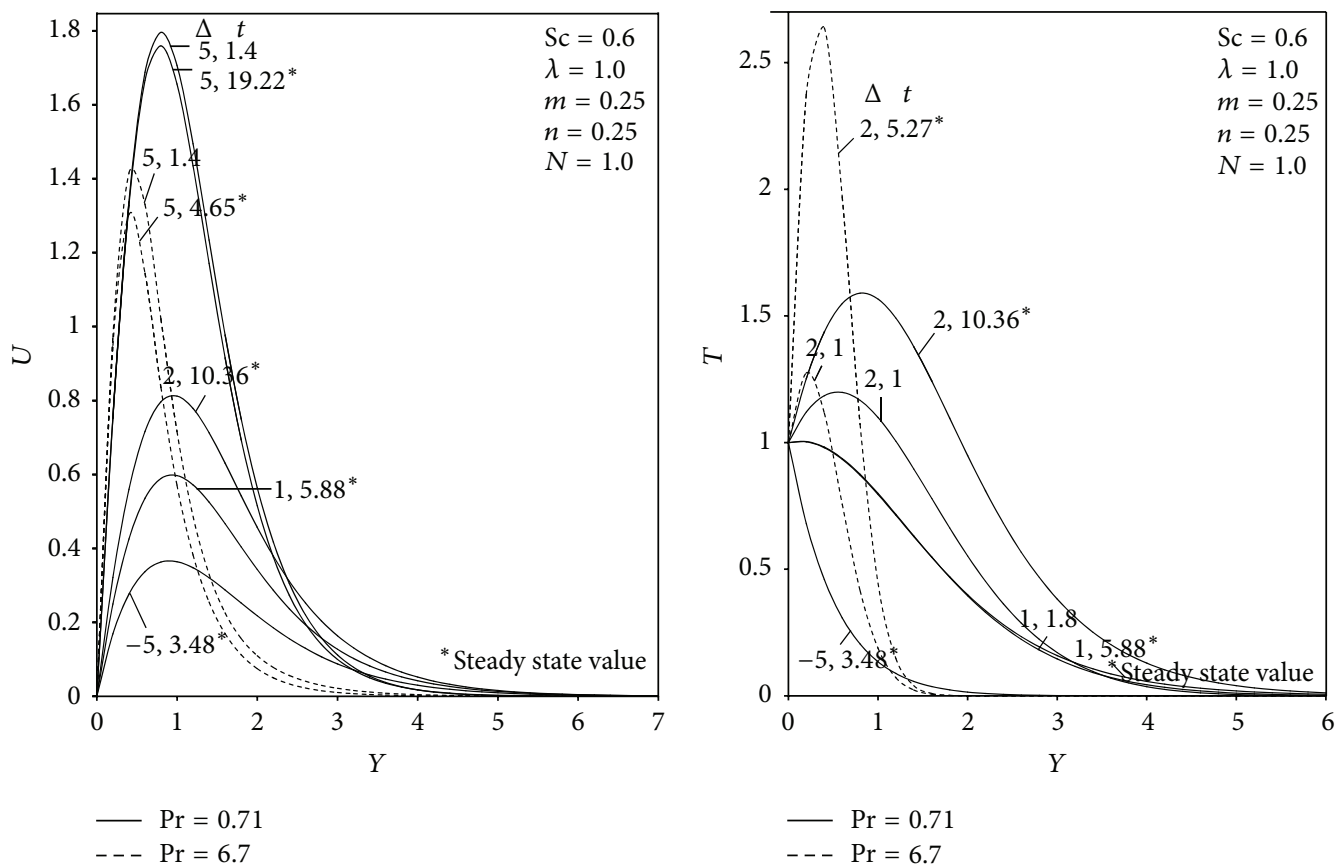

(a)

(b)

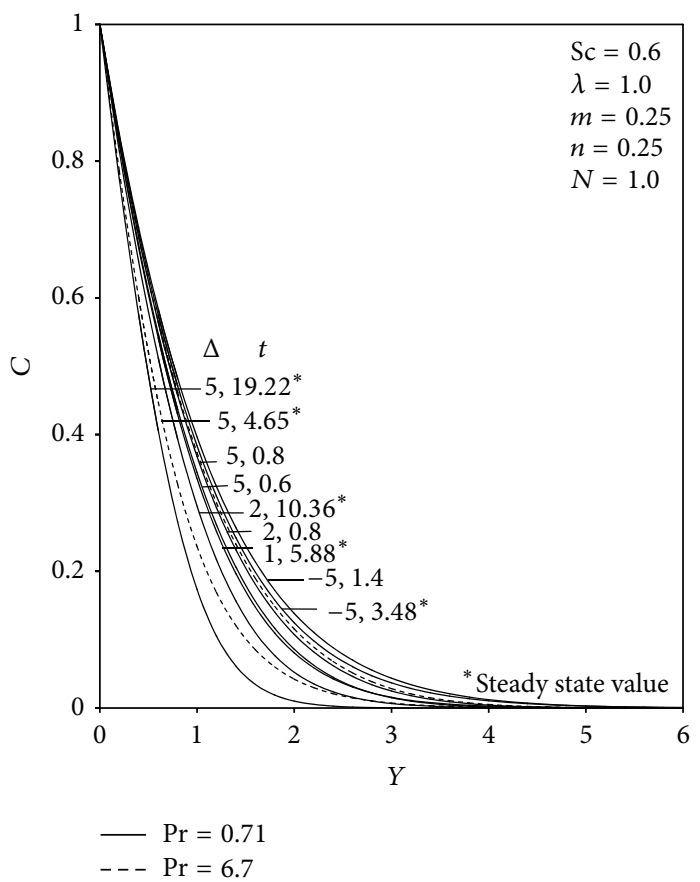

(c)

Figure 2: (a) Transient velocity profiles at $X=1.0$ for different values of $\Delta$ and $\operatorname{Pr}$. (b) Transient temperature profiles at $X=1.0$ for different values of $\Delta$ and Pr. (c) Transient concentration profiles at $X=1.0$ for different values of $\Delta$ and Pr.

to be an inverse linear function of the temperature; the nondimensional nonsimilar governing equations are solved numerically using the fourth-order Runge-Kutta integration scheme with Newton-Raphson shooting technique.

Mohiddin et al. [22, 23] discussed the combined effects of thermal radiation and viscous dissipation on unsteady, laminar, and free convective flow with heat and mass transfer over an incompressible viscous fluid past vertical cone with variable surface temperature and concentration in the presence of a transverse magnetic field applied normal to the surface, heat and mass transfer in a Walters- $B$ viscoelastic fluid along a vertical cone using Crank-Nicholson finite difference 


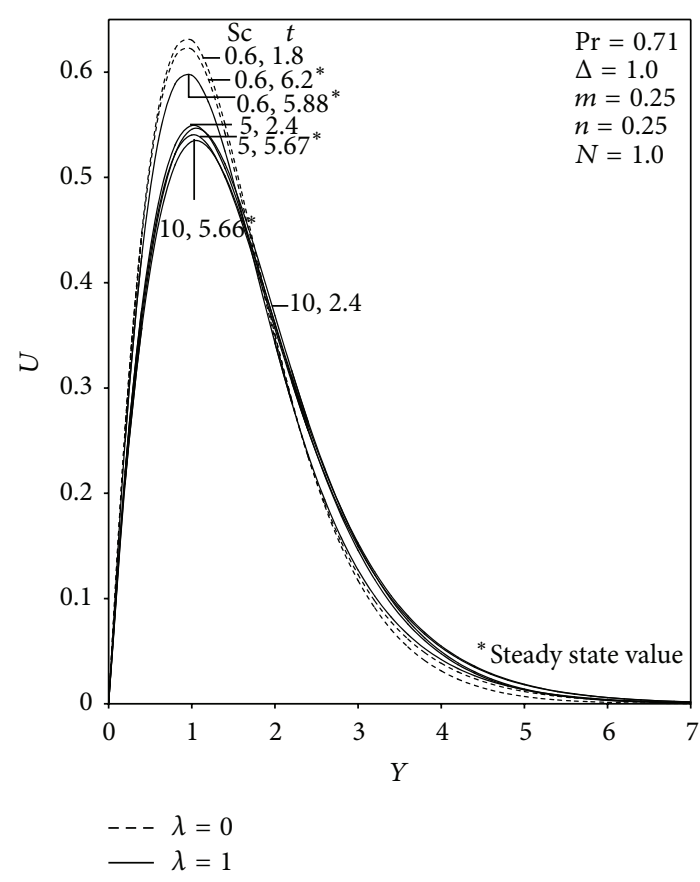

(a)

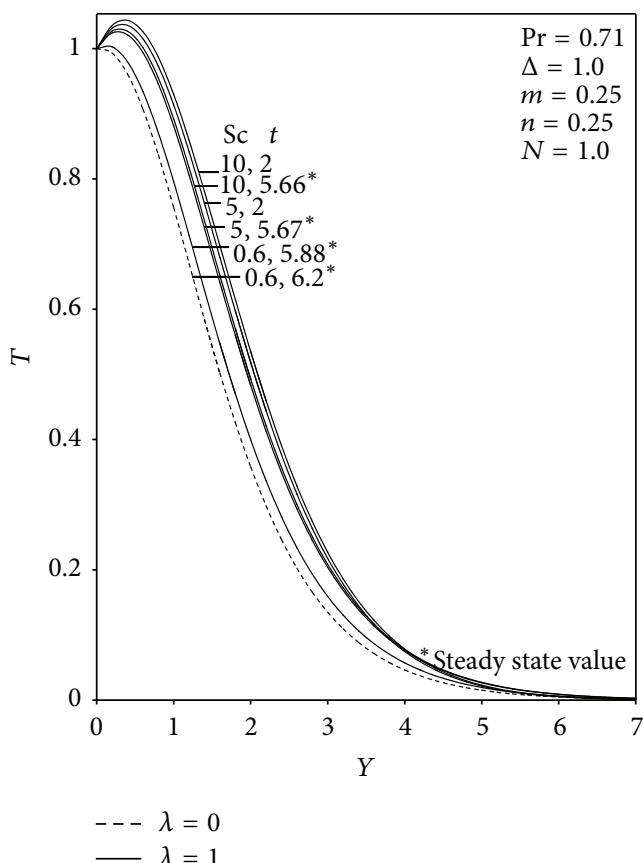

(b)

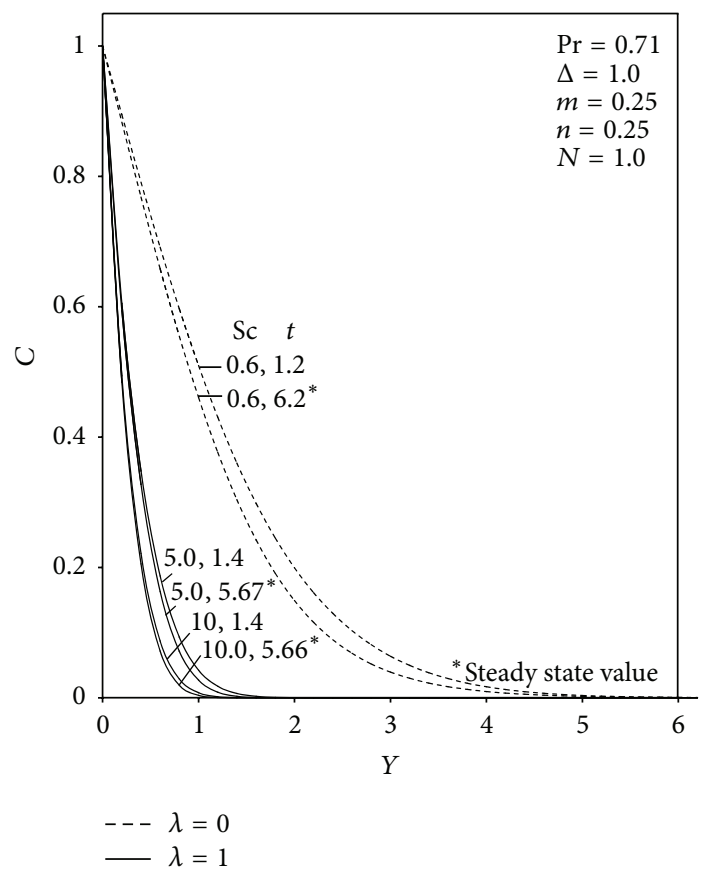

(c)

Figure 3: (a) Transient velocity profiles at $X=1.0$ for different values of $\lambda$ and Sc. (b) Transient temperature profiles at $X=1.0$ for different values of $\lambda$ and Sc. (c) Transient concentration profiles at $X=1.0$ for different values of $\lambda$ and Sc.

scheme. Patil and Pop [24] considered the unsteady mixed convection boundary layer flow over a vertical cone to investigate the combined effects of the buoyancy force, thermal and mass diffusion in the presence of the first-order chemical reaction and surface mass transfer. The governing boundary layer equations are transformed into a nondimensional form by a group of nonsimilar transformations and the resulting system of coupled nonlinear partial differential equations is solved numerically by the combination of quasilinearization technique and an implicit finite difference scheme. Recently El-Kabeir and El-Sayed [25] studied the problem of heat and mass transfer by free convection of a viscoelastic fluid past a vertical isothermal cone surface in the presence of transverse uniform magnetic field and chemical reaction effect taking 


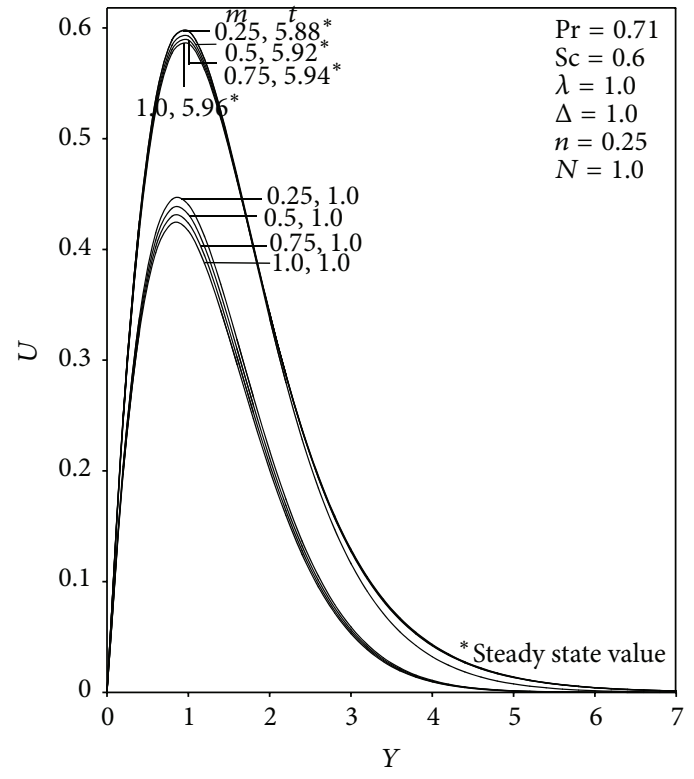

(a)

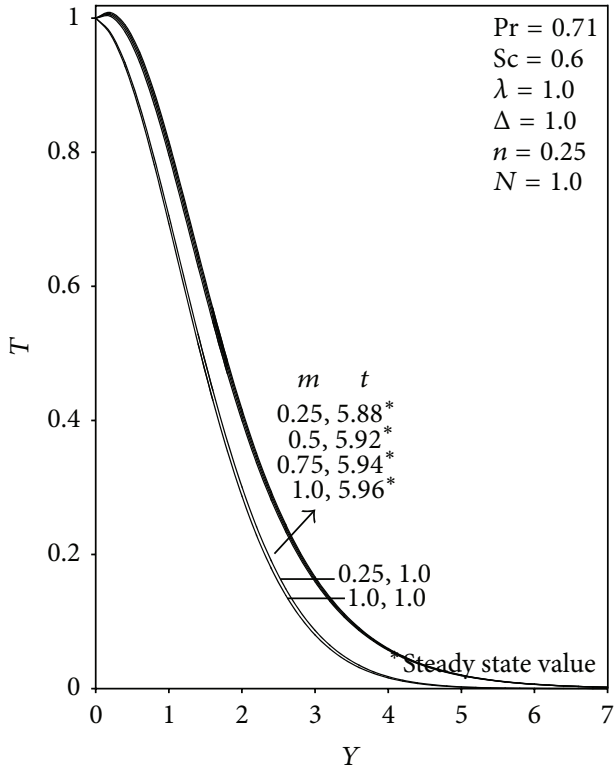

(b)

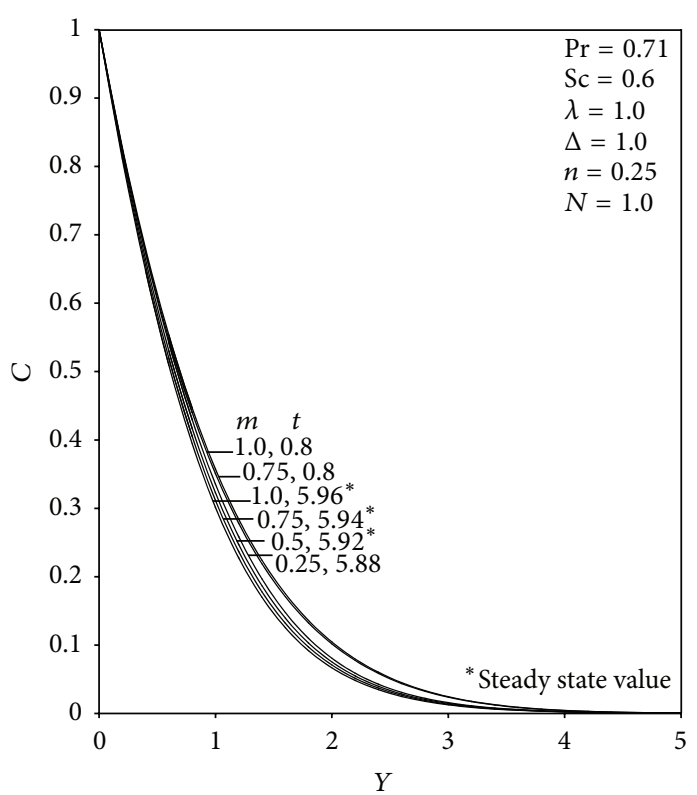

(c)

Figure 4: (a) Transient velocity profiles at $X=1.0$ for different values of $m$. (b) Transient temperature profiles at $X=1.0$ for different values of $m$. (c) Transient concentration profiles at $X=1.0$ for different values of $m$.

into account the effects of viscous dissipation, Joule heating, and thermal radiation. The cone surface is maintained at constant temperature and constant species concentration. The governing partial differential equations are transferred into a system of ordinary differential equations, which are solved numerically using a fourth-order Runge-Kutta scheme with the shooting method. Awad et al. [26] studied the Soret and Dufour effects on the skin friction coefficient, the heat and the mass transfer from an inverted cone in a porous medium. Numerical solutions for the governing momentum, energy, and concentration equations were found using a shooting method together with a sixth-order Runge-Kutta method. The results were validated by using a linearization method. Also Narayana et al. [27] studied the Soret and Dufour effects on free magneto hydrodynamic convection from a vertical spinning cone. They discussed two different types of boundary heating, namely, linear surface temperature (LST), where the surface of the cone is maintained at a temperature that varies linearly with the distance from origin, and linear surface heat flux (LSHF). The nonlinear coupled governing equations were solved using a shooting technique together with a Runge-Kutta method of four slopes. Basiri Parsa et 


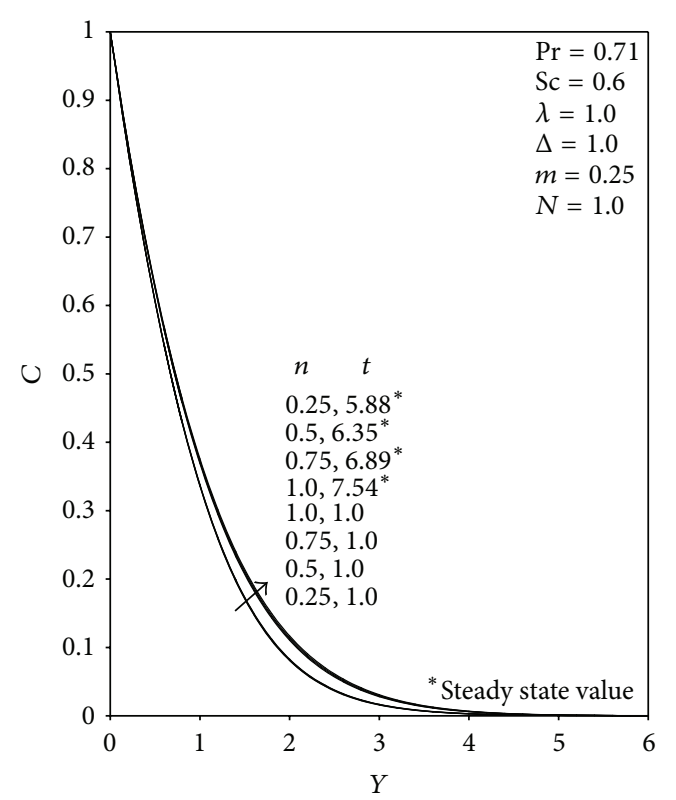

Figure 5: Transient concentration profile at $X=1.0$ for different values of $n$.

al. [28] discussed steady laminar magneto hydrodynamic boundary layer flow past a stretching surface with uniform free stream and internal heat generation or absorption in an electrically conducting fluid. The governing boundary layer and temperature equations for this problem are first transformed into a system of ordinary differential equations using similarity variables and then solved by using a new analytical method and numerical method, by using a fourthorder Runge-Kutta and shooting method.

The objective of the present investigation, namely, transient free convective flow from a nonisothermal vertical cone with heat generation/absorption and chemical reaction, has not received any attention in literature. Also it has a wide range of applications in the field of nuclear reactor safety, solar energy plants, drying, and dehydration process in chemical and food process, design of space crafts and steam generators, and so forth. Hence, the present work studies and deals with the transient free convective flow from a nonisothermal vertical cone with the above said effects. The governing boundary layer equations are solved by an implicit finite difference scheme of Crank-Nicolson type for various values of parameters $\lambda, \Delta, \mathrm{Sc}, \operatorname{Pr}, N, n$, and $m$. In order to check the accuracy of the numerical results, the present results are compared with the available results of Chamkha [8] and they are found to be in excellent agreement.

\section{Mathematical Formulation}

An axisymmetric unsteady, laminar free convection flow of a viscous incompressible fluid past a vertical cone with nonuniform surface temperature and concentration under the influence of chemical reaction and heat generation/absorption is considered. It is assumed that the effects of viscous dissipation and pressure gradient along the boundary layer are negligible.
It is also assumed that there exists first-order chemical reaction between the fluid and the species concentration. The concentration $C^{\prime}$ of the diffusing species is assumed to be very small in comparison to the other chemical species far away from the surface of the cone $C_{\infty}^{\prime}$. Hence the Soret and Dufour effects are neglected. It is also assumed that the cone surface and the surrounding fluid which is at rest are at the same temperature $T_{\infty}^{\prime}$ and concentration $C_{\infty}^{\prime}$. Then at time $t^{\prime}>0$, the temperature of the cone surface is suddenly raised to $T_{w}^{\prime}(x)=T_{\infty}^{\prime}+a x^{n}$ and the concentration near the cone surface is also raised to $C_{w}^{\prime}(x)=C_{\infty}^{\prime}+b x^{m}$ and both are maintained at the same level, where $a, b$ are the positive constants and $n, m$ are the exponents in power law variation in surface temperature and concentration, respectively. The coordinate system is chosen (as shown in Figure 1) such that $x$ measures the distance along surface of the cone from the apex $(x=0)$ and $y$ measures the distance normally outward. The fluid properties are assumed to be constant except the density variations causing a body force in the momentum equation. The governing boundary layer equations of continuity, momentum, energy, and concentration under Boussinesq approximation are as follows: equation of continuity:

$$
\frac{\partial}{\partial x}(r u)+\frac{\partial}{\partial y}(r v)=0
$$

equation of momentum:

$$
\begin{aligned}
\frac{\partial u}{\partial t^{\prime}}+ & u \frac{\partial u}{\partial x}+v \frac{\partial u}{\partial y} \\
= & g \beta\left(T^{\prime}-T_{\infty}^{\prime}\right) \cos \phi+v \frac{\partial^{2} u}{\partial y^{2}} \\
& +g \beta^{*}\left(C^{\prime}-C_{\infty}^{\prime}\right) \cos \phi ;
\end{aligned}
$$

equation of energy:

$$
\frac{\partial T^{\prime}}{\partial t^{\prime}}+u \frac{\partial T^{\prime}}{\partial x}+v \frac{\partial T^{\prime}}{\partial y}=\alpha \frac{\partial^{2} T^{\prime}}{\partial y^{2}}+\frac{Q_{o}}{\rho c_{p}}\left(T^{\prime}-T_{\infty}\right)
$$

equation of concentration:

$$
\frac{\partial C^{\prime}}{\partial t^{\prime}}+u \frac{\partial C^{\prime}}{\partial x}+v \frac{\partial C^{\prime}}{\partial y}=D \frac{\partial^{2} C^{\prime}}{\partial y^{2}}-k_{1}\left(C^{\prime}-C_{\infty}\right) .
$$

The initial and boundary conditions are

$$
\begin{gathered}
t^{\prime} \leq 0: \quad u=0, \quad v=0, \\
T^{\prime}=T_{\infty}^{\prime}, \quad C^{\prime}=C_{\infty}^{\prime} \quad \forall x, y, \\
t^{\prime}>0: \quad u=0, \quad v=0, \\
T^{\prime}(x)=T_{\infty}^{\prime}+a x^{n}, \\
C^{\prime}(x)=C_{\infty}^{\prime}+b x^{m} \quad \text { at } y=0, \\
u=0, \quad T^{\prime}=T_{\infty}^{\prime}, \quad C^{\prime}=C_{\infty}^{\prime} \quad \text { at } x=0, \\
u \longrightarrow 0, \quad T^{\prime} \longrightarrow T_{\infty}^{\prime}, \quad C^{\prime} \longrightarrow C_{\infty}^{\prime} \quad \text { as } y \longrightarrow \infty .
\end{gathered}
$$




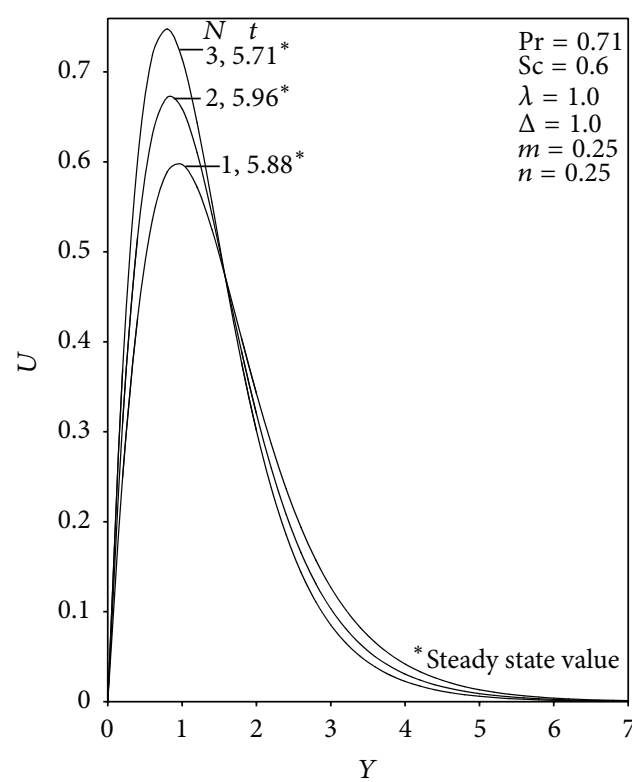

(a)

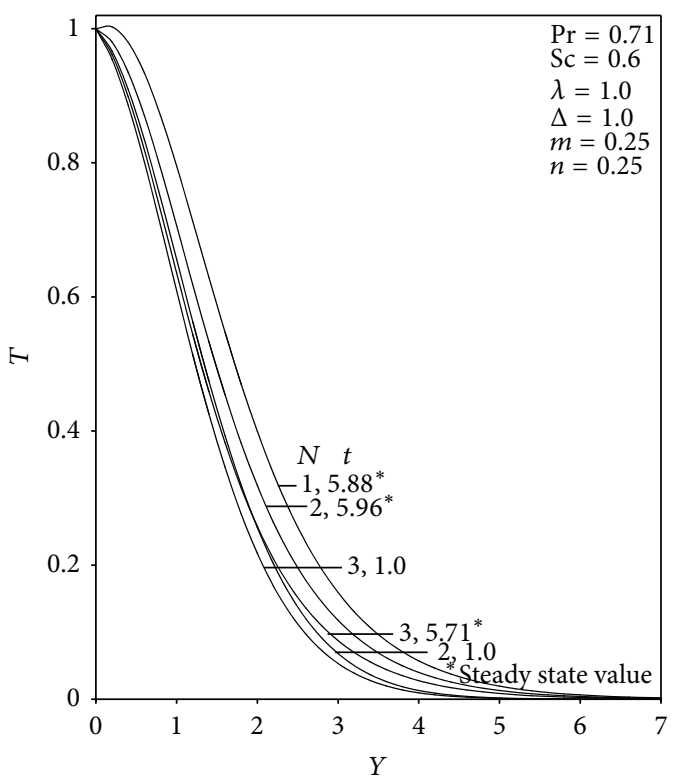

(b)

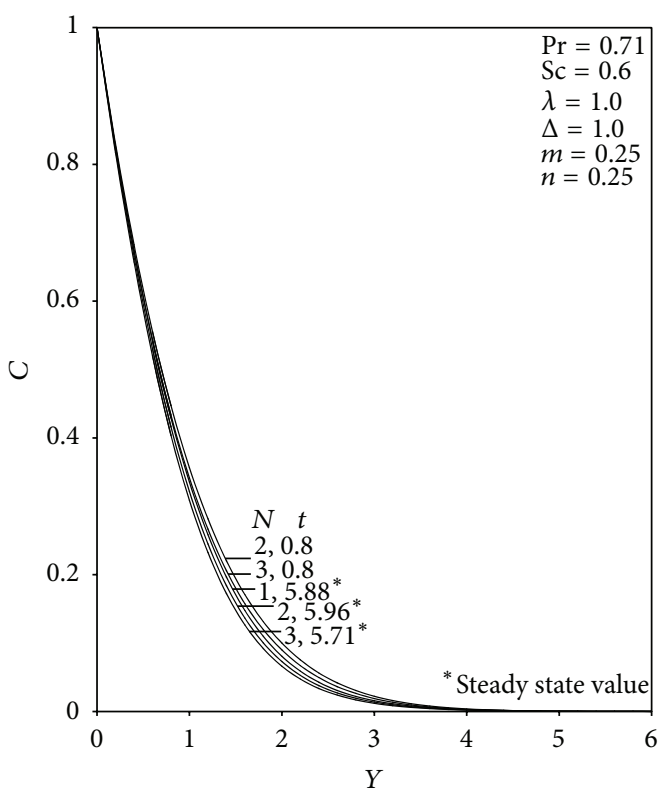

(c)

Figure 6: (a) Transient velocity profiles at $X=1.0$ for different values of $N$. (b) Transient temperature profiles at $X=1.0$ for different values of $N$. (c) Transient concentration profiles at $X=1.0$ for different values of $N$.

Local skin friction, local Nusselt number, and local Sherwood number are given by

$$
\begin{gathered}
\tau_{x}=\mu\left(\frac{\partial u}{\partial y}\right)_{y=0}, \\
\mathrm{Nu}_{x}=\frac{-x\left(\partial T^{\prime} / \partial y\right)_{y=0}}{T_{w}^{\prime}-T_{\infty}^{\prime}}, \\
\mathrm{Sh}_{x}=\frac{-x\left(\partial C^{\prime} / \partial y\right)_{y=0}}{C_{w}^{\prime}-C_{\infty}^{\prime}} .
\end{gathered}
$$

Using the following nondimensional quantities:

$$
\begin{aligned}
& X=\frac{x}{L}, \quad Y=\frac{y}{L}\left(\mathrm{Gr}_{L}\right)^{1 / 4}, \quad R=\frac{r}{L}, \\
& \text { where } r=x \sin \phi, \\
& V=\frac{v L}{v}\left(\mathrm{Gr}_{L}\right)^{-1 / 4}, \quad U=\frac{u L}{v}\left(\mathrm{Gr}_{L}\right)^{-1 / 2}, \\
& t=\frac{v t^{\prime}}{L^{2}}\left(\mathrm{Gr}_{L}\right)^{1 / 2}, \\
& T=\frac{\left(T^{\prime}-T_{\infty}^{\prime}\right)}{\left(T_{w}^{\prime}-T_{\infty}^{\prime}\right)}, \quad \mathrm{Gr}_{L}=\frac{g \beta\left(T_{w}^{\prime}-T_{\infty}^{\prime}\right) L^{3} \cos \phi}{v^{2}},
\end{aligned}
$$



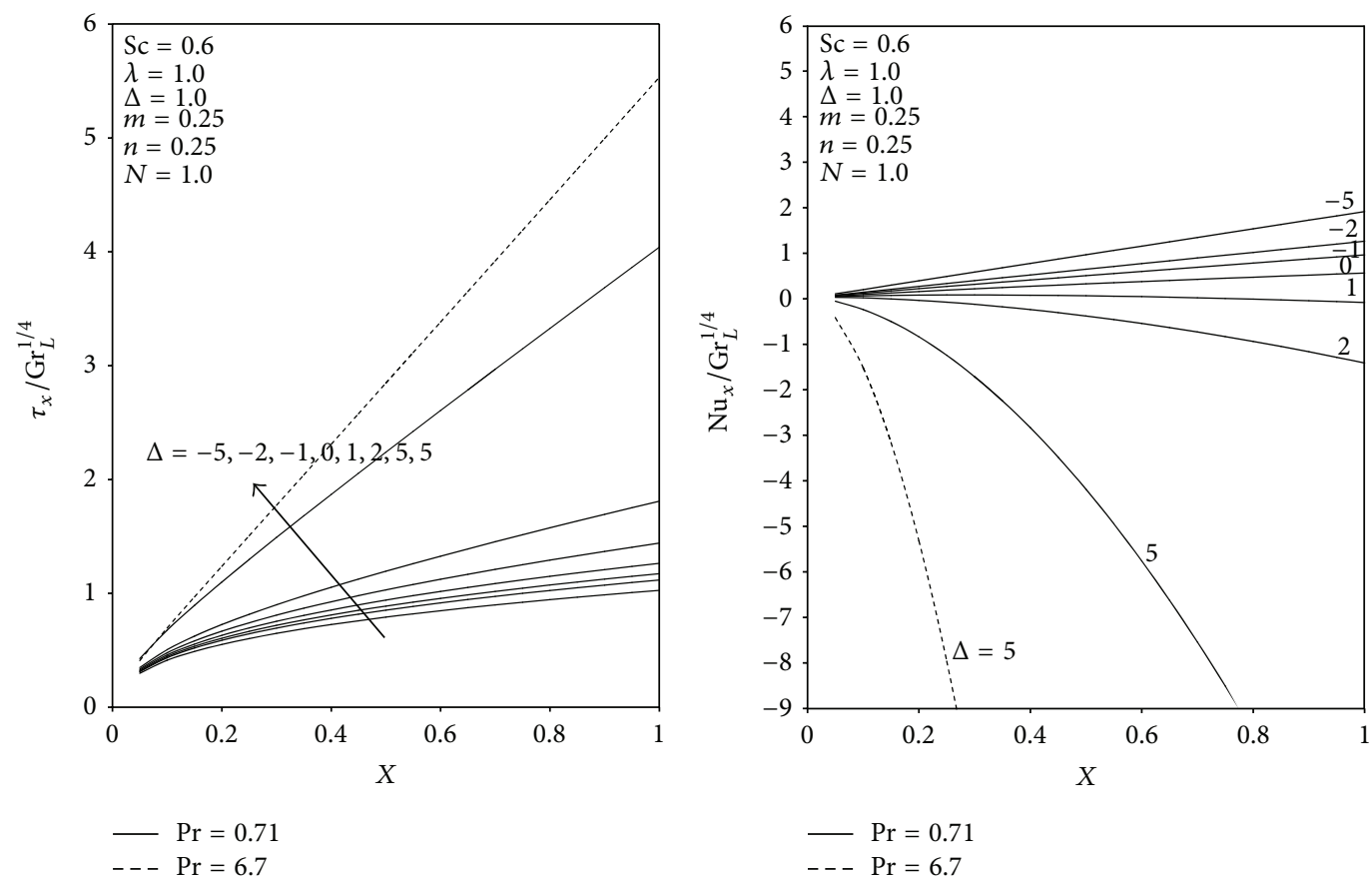

(a)

(b)

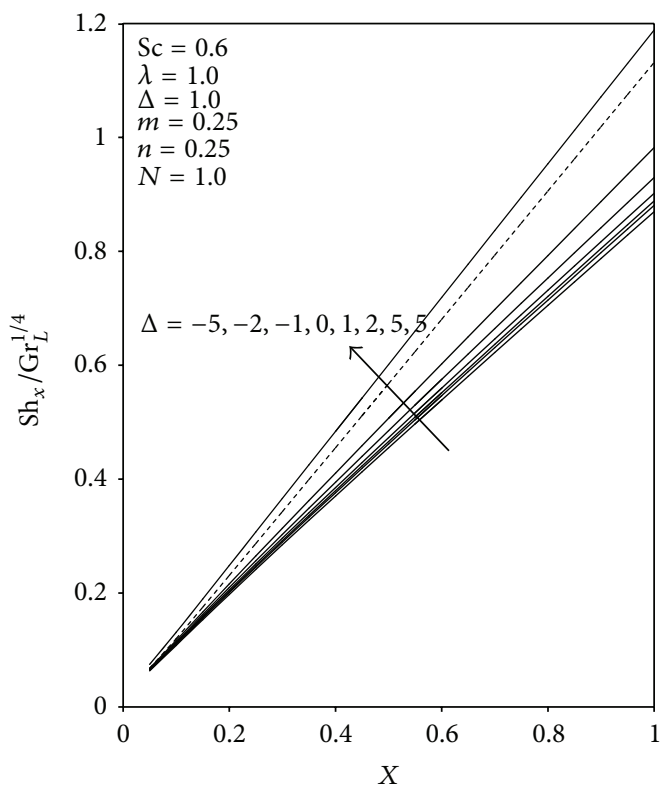

$-\operatorname{Pr}=0.71$

(c)

FIGURE 7: (a) Local skin friction for different values of $\Delta$ and Pr. (b) Local Nusselt number for different values of $\Delta$ and Pr. (c) Local Sherwood number for different values of $\Delta$ and Pr.

$$
\begin{array}{ll}
\operatorname{Pr}=\frac{v}{\alpha}, & \mathrm{Sc}=\frac{v}{D}, \quad N=\frac{\mathrm{Gr}^{*}}{\mathrm{Gr}_{L}}, \quad \Delta=\frac{Q_{o} L^{2}}{C_{p} \mu}\left(\mathrm{Gr}_{L}\right)^{-1 / 2}, \\
T=\frac{\left(C^{\prime}-C_{\infty}^{\prime}\right)}{\left(C_{w}^{\prime}-C_{\infty}^{\prime}\right)}, \quad \mathrm{Gr}^{*}=\frac{g \beta^{*}\left(C_{w}^{\prime}-C_{\infty}^{\prime}\right) L^{3} \cos \phi}{v^{2}}, \quad \lambda=\frac{k_{1} L^{2}}{v}\left(\mathrm{Gr}_{L}\right)^{-1 / 2} .
\end{array}
$$




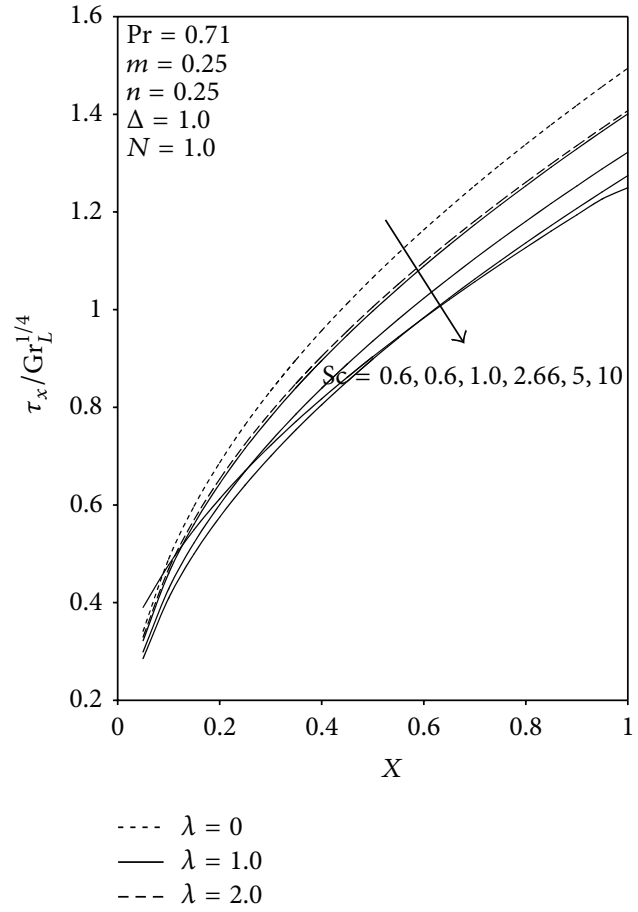

(a)

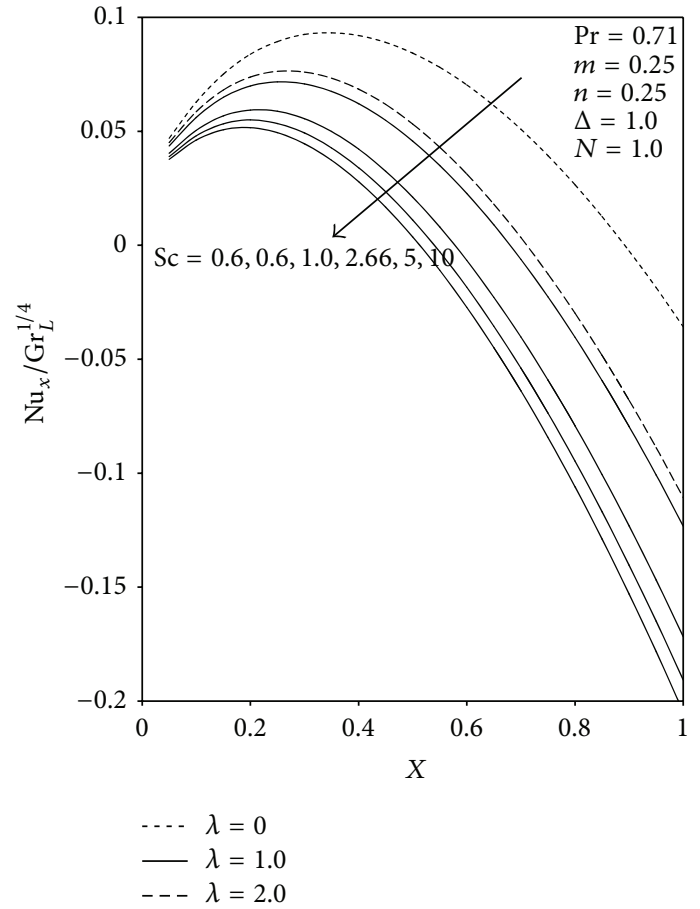

(b)

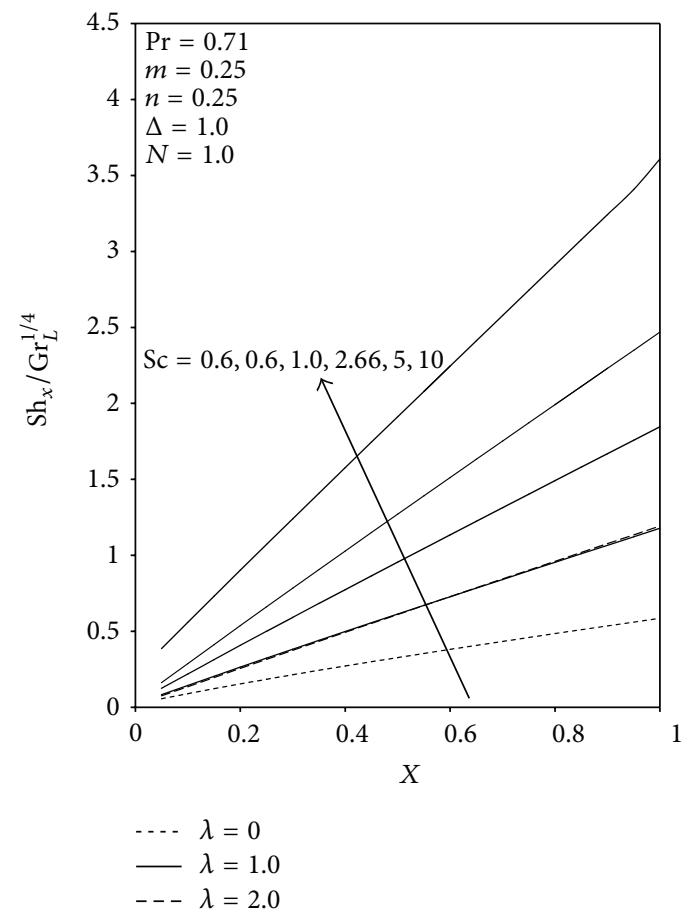

(c)

Figure 8: (a) Local skin friction profiles for different values of $\lambda$ and Sc. (b) Local Nusselt number profiles for different values of $\lambda$ and Sc. (c) Local Sherwood number profiles for different values of $\lambda$ and Sc.

Equations (1), (2), (3), (4), and (5) can then be written in the following nondimensional form:

$$
\frac{\partial(U R)}{\partial X}+\frac{\partial(V R)}{\partial Y}=0
$$

$$
\begin{gathered}
\frac{\partial U}{\partial t}+U \frac{\partial U}{\partial X}+V \frac{\partial U}{\partial Y}=T+N C+\frac{\partial^{2} U}{\partial Y^{2}} \\
\frac{\partial T}{\partial t}+U \frac{\partial T}{\partial X}+V \frac{\partial T}{\partial Y}=\frac{1}{\operatorname{Pr}} \frac{\partial^{2} T}{\partial Y^{2}}+\Delta T
\end{gathered}
$$




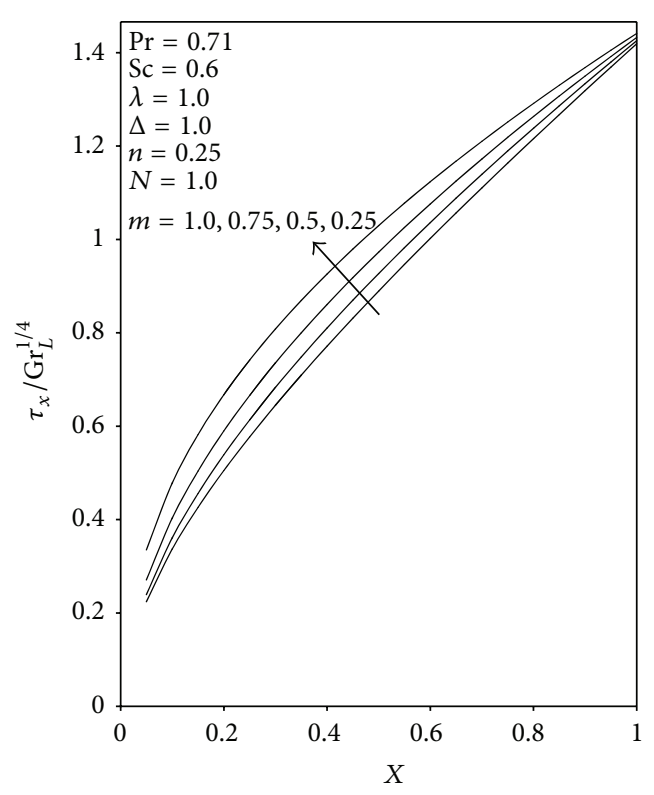

(a)

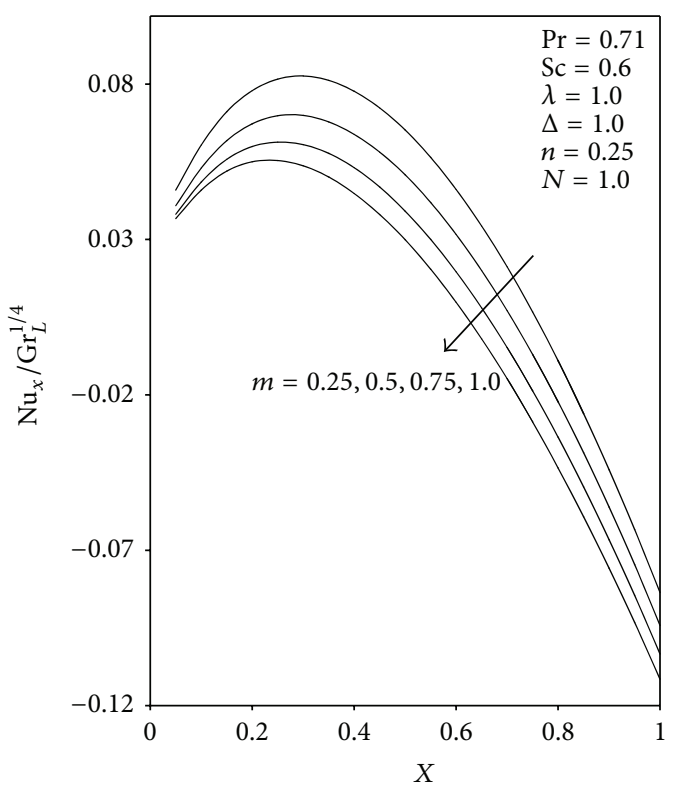

(b)

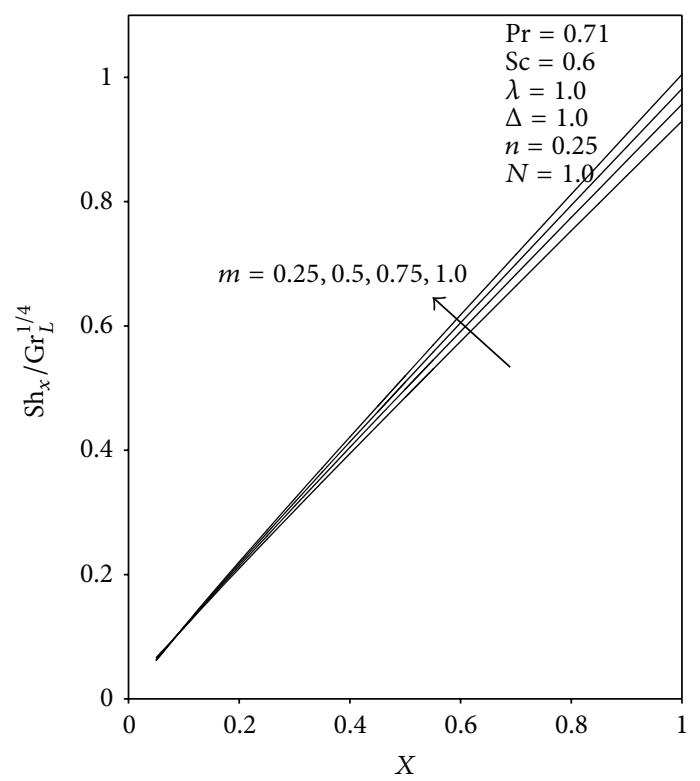

(c)

FiguRE 9: (a) Local skin friction profiles for different values of $m$. (b) Local Nusselt number profiles for different values of $m$. (c) Local Sherwood number profiles for different values of $m$.

$$
\frac{\partial C}{\partial t}+U \frac{\partial C}{\partial X}+V \frac{\partial C}{\partial Y}=\frac{1}{S c} \frac{\partial^{2} C}{\partial Y^{2}}-\lambda C
$$

The corresponding nondimensional initial and boundary conditions are

$$
\begin{gathered}
t \leq 0: \quad U=0, \quad V=0, \\
T=0, \quad C=0 \quad \forall X, Y, \\
t>0: \quad U=0, \quad V=0, \quad T=X^{n},
\end{gathered}
$$

$$
\begin{gathered}
C=X^{m} \quad \text { at } Y=0, \\
U=0, \quad T=0, \quad C=0 \quad \text { at } X=0, \\
U \longrightarrow 0, \quad T \longrightarrow 0, \quad C \longrightarrow 0 \quad \text { as } Y \longrightarrow \infty .
\end{gathered}
$$

Local skin friction, local Nusselt number, and local Sherwood number in nondimensional quantities are

$$
\tau_{X}=\mathrm{Gr}_{L}^{3 / 4}\left(\frac{\partial U}{\partial Y}\right)_{Y=0},
$$




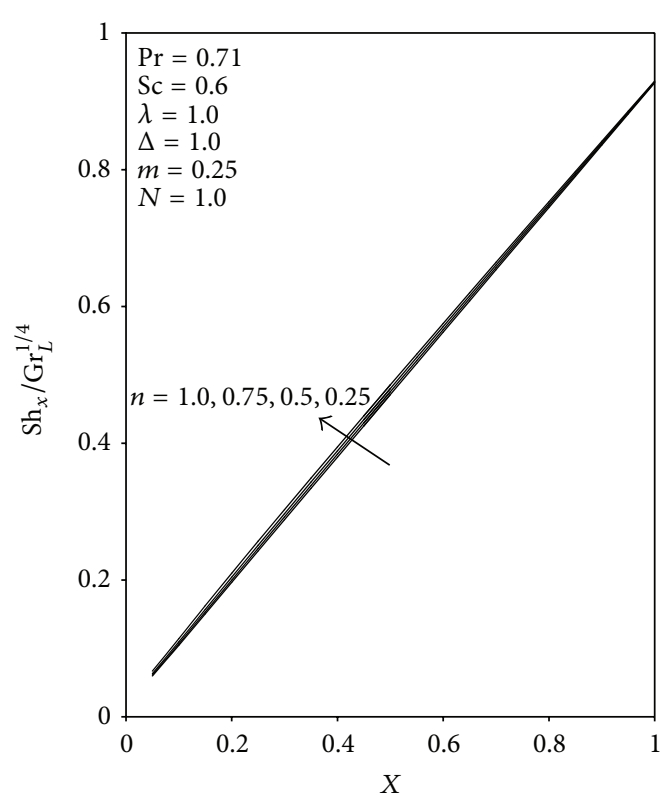

FIGURE 10: Local Sherwood number profiles for different values of $n$.

$$
\begin{aligned}
\mathrm{Nu}_{X} & =\frac{X}{T_{Y=0}}\left(\frac{-\partial T}{\partial Y}\right)_{Y=0} \mathrm{Gr}_{L}^{1 / 4} \\
\mathrm{Sh}_{X} & =\frac{X}{C_{Y=0}}\left(\frac{-\partial C}{\partial Y}\right)_{Y=0} \mathrm{Gr}_{L}^{1 / 4}
\end{aligned}
$$

Average skin friction, average Nusselt number, and average Sherwood number in nondimensional quantities are

$$
\begin{aligned}
\bar{\tau} & =2 \mathrm{Gr}_{L}^{3 / 4} \int_{0}^{1} X\left(\frac{\partial U}{\partial Y}\right)_{Y=0} d X, \\
\overline{\mathrm{Nu}} & =2 \mathrm{Gr}_{L}^{1 / 4} \int_{0}^{1} \frac{X}{T_{Y=0}}\left(\frac{-\partial T}{\partial Y}\right)_{Y=0} d X \\
\overline{\mathrm{Sh}} & =2 \mathrm{Gr}_{L}^{1 / 4} \int_{0}^{1} \frac{X}{C_{Y=0}}\left(\frac{-\partial C}{\partial Y}\right)_{Y=0} d X .
\end{aligned}
$$

\section{Solution Procedure}

The unsteady nonlinear coupled partial differential equations (8) with the initial and boundary conditions (9) are solved by employing a finite difference scheme of Crank-Nicholson type which is rapidly convergent and unconditionally stable as discussed by Soundalgekar and Ganesan [29], Bapuji et al. [30], and Muthucumaraswamy and Ganesan [31, 32]. The region of integration is considered as a rectangle with $X_{\max }(=1)$ and $Y_{\max }(=20)$, where $Y_{\max }$ corresponds to $Y=\infty$ which lies very well outside both the momentum and thermal boundary layers. The maximum of $Y$ was chosen as 20, after some preliminary investigation so that the last two boundary conditions of (9) are satisfied within the tolerance limit of $10^{-5}$. The mesh sizes have been fixed as $\Delta X=0.05, \Delta Y=0.05$ with time step $\Delta t=0.01$. The computations are carried out first by reducing the spatial mesh sizes by $50 \%$ in one direction and later in both directions by $50 \%$. The results are compared. It is observed in all cases that the results differ only in the fifth decimal place. Hence, the choice of the mesh sizes seems to be appropriate. The scheme is unconditionally stable as described by Bapuji et al. [33]. The local truncation error is $O\left(\Delta t^{2}+\Delta Y^{2}+\Delta X\right)$ and it tends to zero as $\Delta t, \Delta Y$, and $\Delta X$ tend to zero. Hence, the scheme is compatible. Stability and compatibility ensures the convergence.

\section{Result and Discussion}

In order to prove the accuracy of our numerical results, the present results for the steady state flow at $X=1.0$ are compared with available solutions from the open literature. The numerical values of the local skin friction $\tau_{X}$ and the local Nusselt number $\mathrm{Nu}_{X}$ for different values of the Prandtl number with $M=0, N=0, n=0$, and $R_{d}=0$ are compared with the results of Chamkha [8] in Table 1 , where $f^{\prime \prime}(\infty, 0)$ and $-\theta^{\prime}(\infty, 0)$ are the steady state local skin friction and the local Nusselt number for a full cone. It is observed that the results are in good agreement with each other.

Velocity, temperature, and concentration profiles for different values of Prandtl number Pr, heat generation/absorption parameter $\Delta$, are shown in Figures $2(\mathrm{a})-2(\mathrm{c})$. The positive values of $\Delta$ represent the presence of heat generation and the negative values correspond to heat absorption. It is noted from Figure 2(a) that the effect of the heat generation/ absorption parameter $\Delta$ on the velocity distribution. It is seen from the figure that when heat is generated the buoyancy force increases which induces the flow rate to increase and giving rise to the velocity profiles but the momentum boundary layer decreases as $\Delta$ and $\operatorname{Pr}$ increases. Figure 2(b) depicts the temperature increases for higher values of $\Delta$ and lower values of Pr; the thermal boundary layer decreases for the larger value of $\Delta$ and $\operatorname{Pr}$. From Figure 2(c) it is seen that the concentration decreases and the time taken to reach the steady state is increased when $\Delta$ and Pr increase. Also the concentration boundary layer becomes thin for higher values of $\Delta$ and $\operatorname{Pr}$.

Figures 3(a)-3(c) depict the transient velocity, temperature, and concentration profiles for various values of chemical reaction parameter $\lambda$ and Schmidt number Sc. As the Schmidt number increases the concentration decreases. This causes the concentration buoyancy effects to decrease. The velocity and boundary layer thickness decreases with an increase in $\lambda$ and Sc (Figure 3(a)), whereas the temperature increases for larger values of $S c$ and smaller values of $\lambda$ and the thermal boundary layer becomes thin for smaller values of Sc and $\lambda$ (Figure 3(b)). Figure 3(c) shows that the concentration decreases for lower values of $\lambda$ and higher values of Sc. With increasing Sc the velocity is depressed through the boundary layer; that is, the flow is retarded. Higher Sc values will physically correspond to a decrease of molecular diffusivity of the primary fluid causing a decrease in the rate of species diffusion. Lower Sc values will exert the reverse influence since they correspond to higher molecular diffusivities. Concentration boundary layer thickness is therefore considerably greater for $\mathrm{Sc}=0.6$ than for $\mathrm{Sc}=10$. 


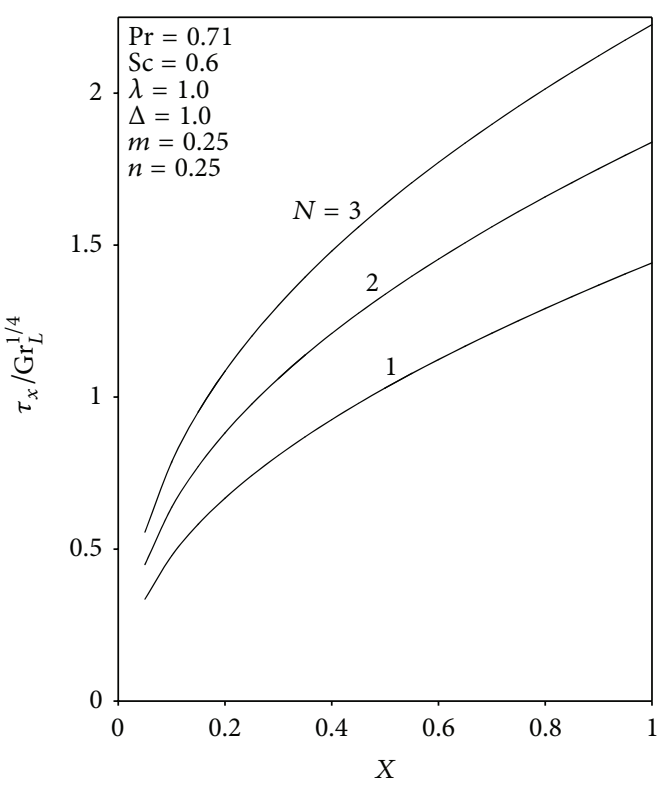

(a)

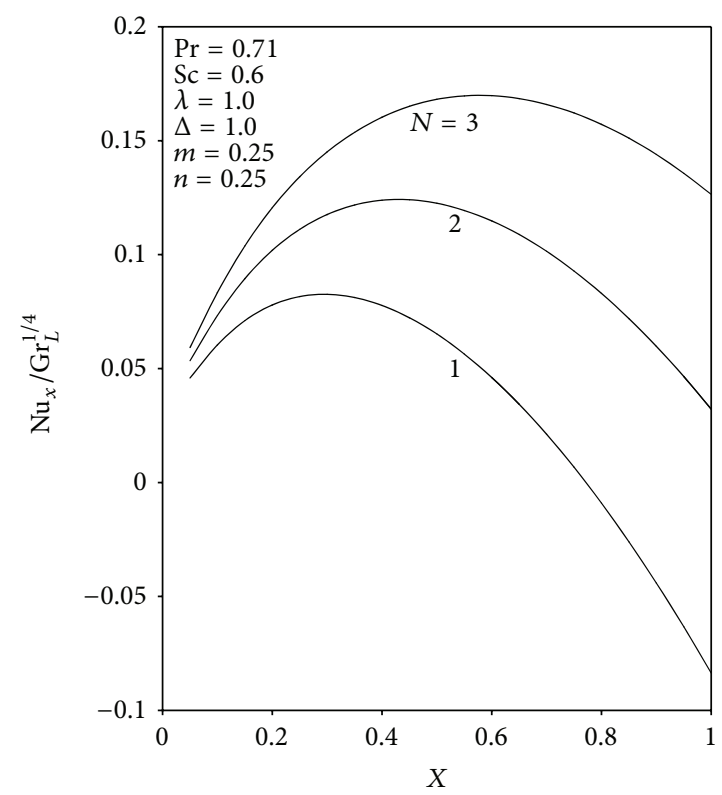

(b)

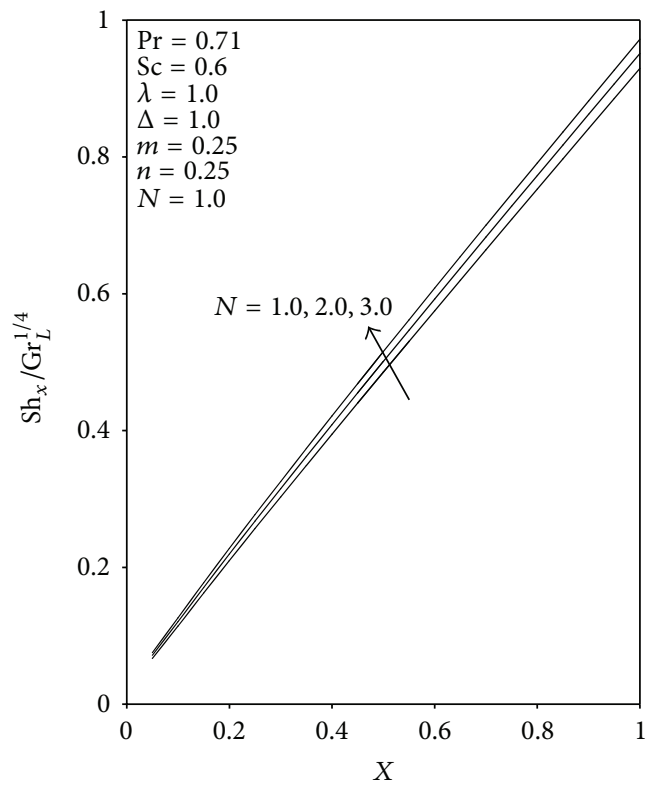

(c)

Figure 11: (a) Local skin friction profiles for different values of $N$. (b) Local Nusselt number profiles for different values of $N$. (c) Local Sherwood number profiles for different values of $N$.

Figures 4(a)-4(c) show the influence of the surface concentration power law exponent $m$ on velocity, temperature, and concentration distributions. We observe in Figure 4(a) that the velocity is maximized throughout the boundary layer with a decrease in $m$. Figure 4(b) indicates that the temperature increases for smaller values of $m$. As such increasing power law exponents in the cone surface concentration variations serve to decelerate the flow in the boundary layer. Concentration of the species increases for smaller values of $m$ and larger values of $n$ is observed from Figures 4(c) and
5. The effects of the buoyancy ratio parameter $N$ on the transient velocity, temperature, and concentration profiles are shown in Figures 6(a)-6(c). The velocity increases steadily with time reaches a temporal maximum and consequently it reaches the steady state. However, time required to reach the steady state depends upon buoyancy ratio parameter $N$. An increase in $N$ leads to an increase in the velocity; that is, as $N$ increases, the combined buoyancy force also increases; therefore, the velocity increases near the surface of the cone (Figure 6(a)). As we move away from the surface 


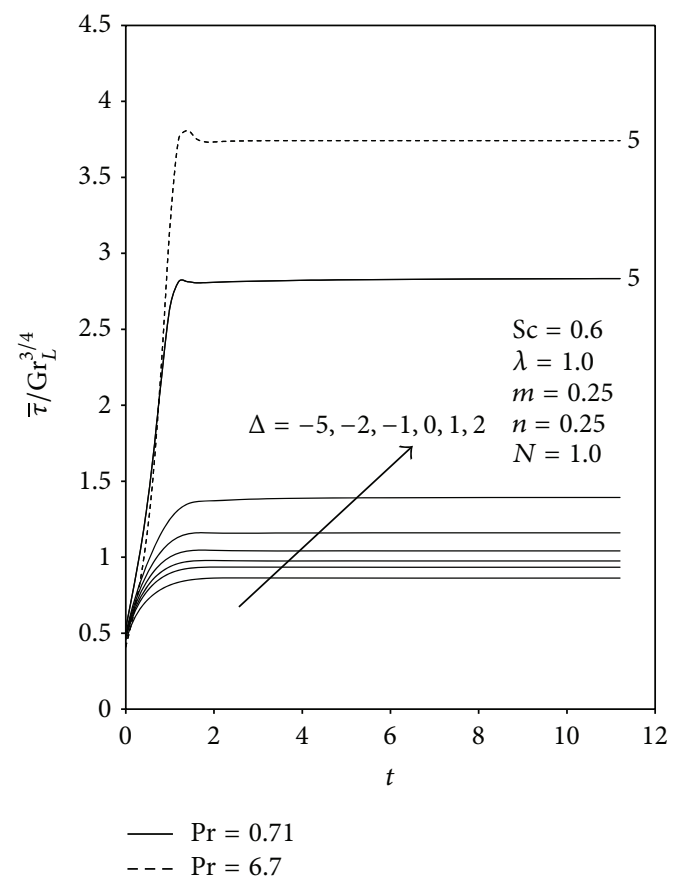

(a)

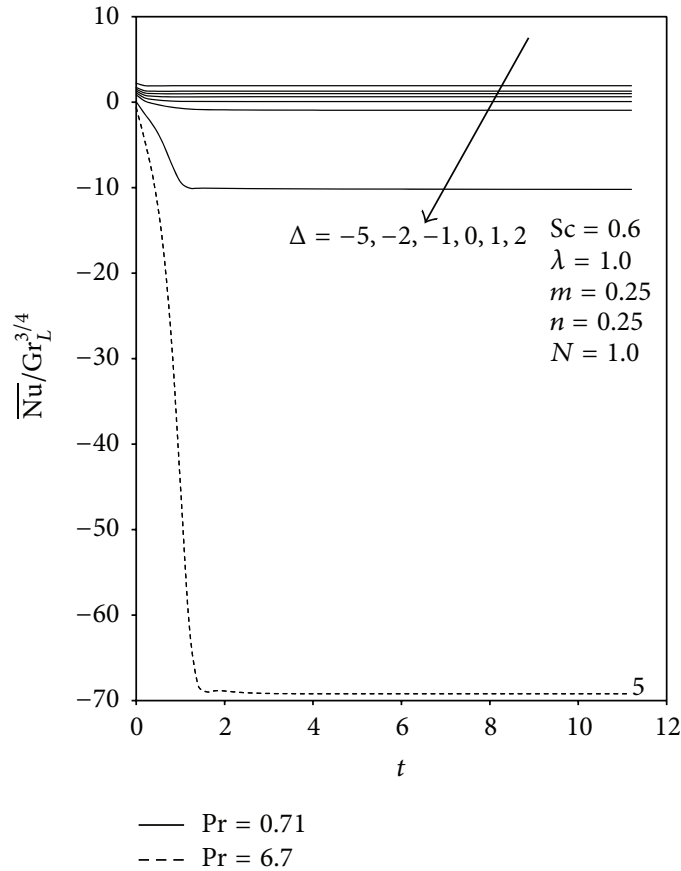

(b)

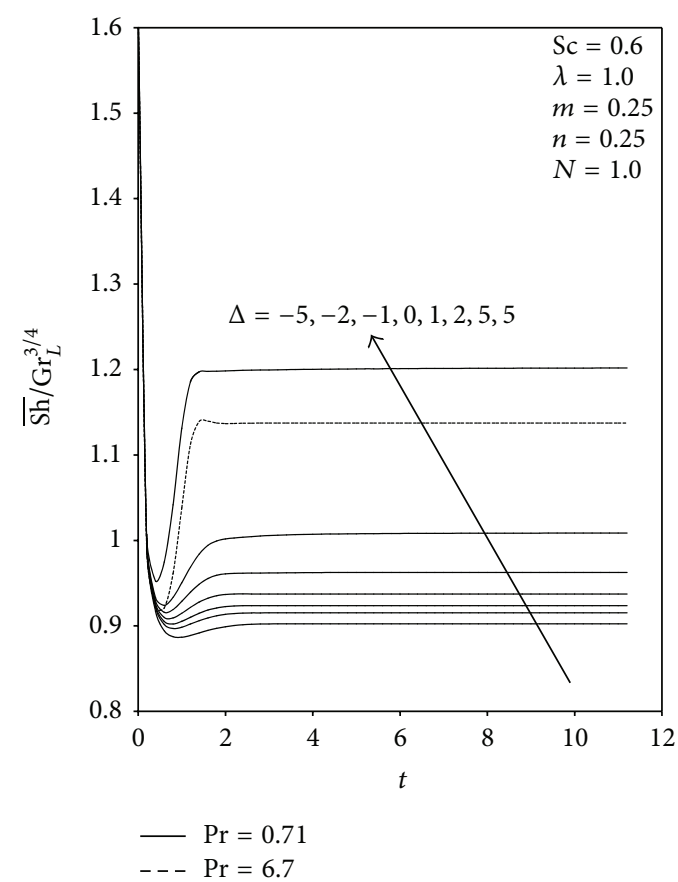

(c)

Figure 12: (a) Average skin friction profiles for different values of $\Delta$ and $\operatorname{Pr}$ in transient state. (b) Average Nusselt number profiles for different values of $\Delta$ and $\operatorname{Pr}$ in transient state. (c) Average Sherwood number profiles for different values of $\Delta$ and $\operatorname{Pr}$ in transient state.

of the cone, the temperature decreases for all the values of $N$ (Figure 6(b)); thus for higher value of buoyancy ratio parameter $N$ the fluid cools rapidly and concentration field decreases with increasing value of buoyancy ratio parameter $N$ (Figure 6(c)).

Local values of the skin friction $\tau_{X}$, Nusselt number $\mathrm{Nu}_{X}$, and Sherwood number $\mathrm{Sh}_{X}$ for different parameters
$\operatorname{Pr}, \Delta, \lambda, \mathrm{Sc}, m, n$, and $N$ are plotted through Figures 7 (a)$7(\mathrm{c})$ to Figures 11(a)-11(c). Figures 7(a)-7(c) indicate that the local skin friction increases for larger values of $\Delta$ and $\operatorname{Pr}$ (Figure 7(a)). The local Nusselt number increases for smaller values of $\Delta$ and larger values of $\operatorname{Pr}$; that is, the heat generation/absorption parameter $\Delta$ has the tendency to increase the magnitude of the local Nusselt number for $\Delta<0$ 

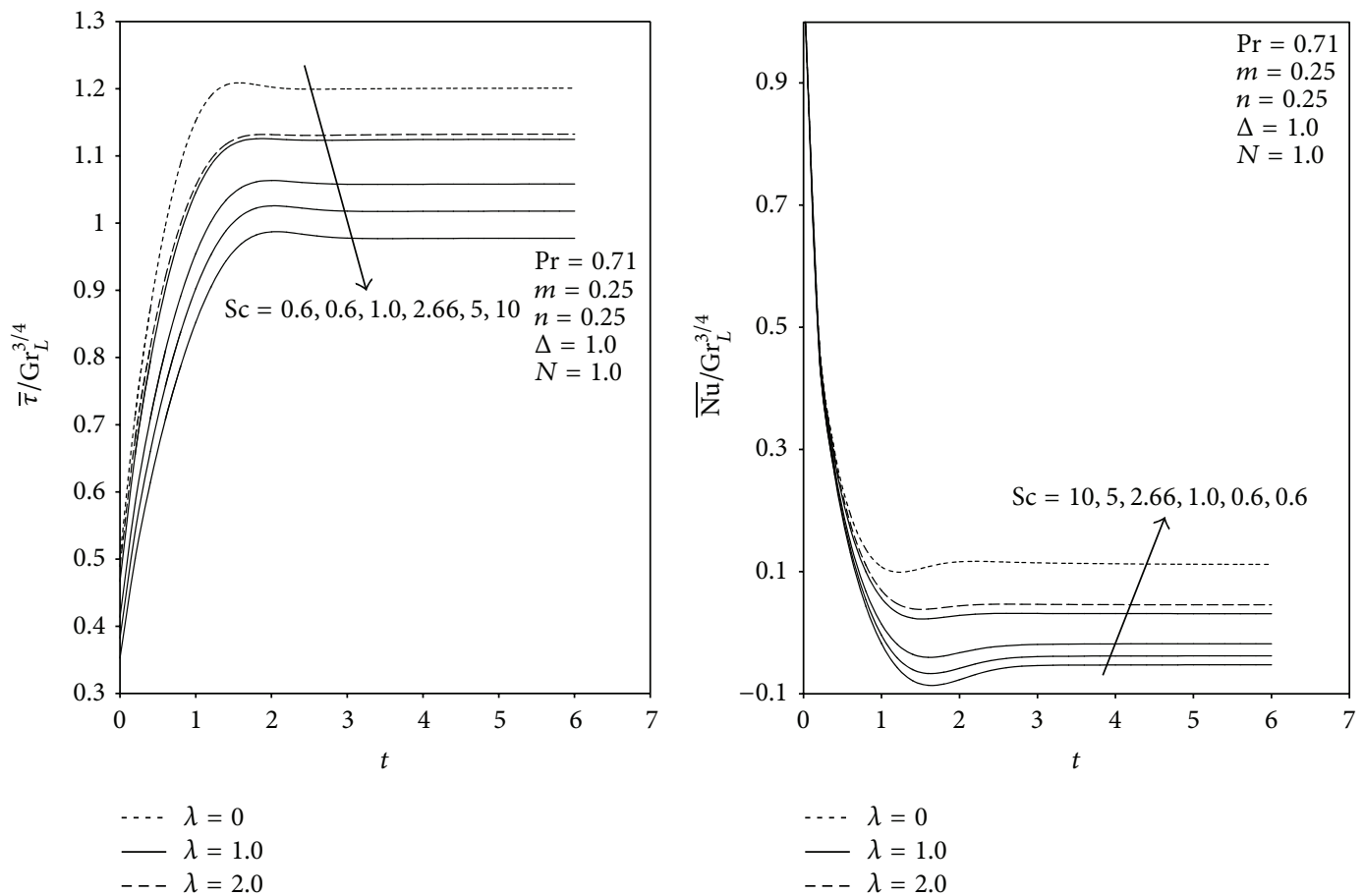

(a)

(b)

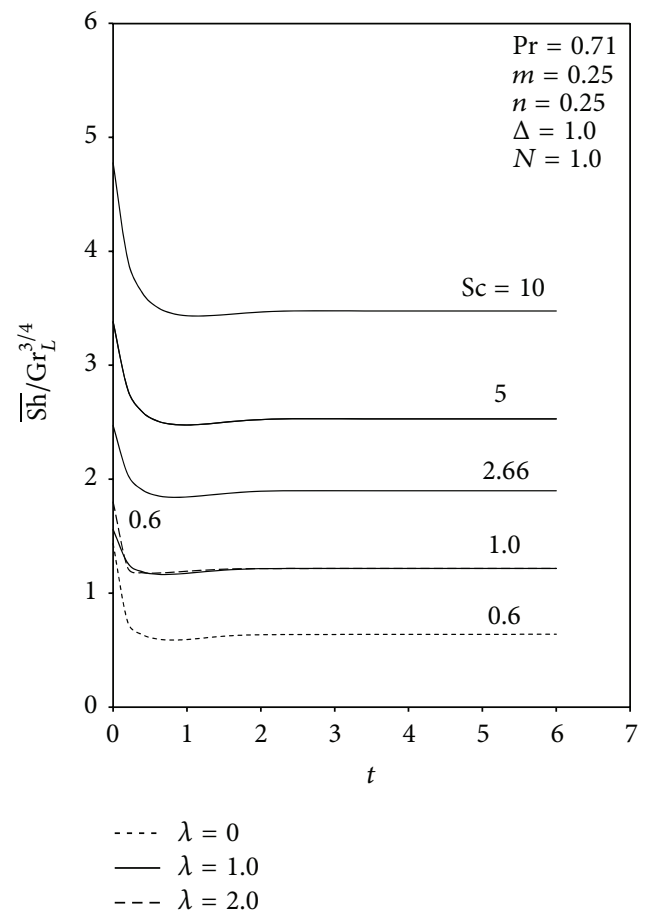

(c)

FiguRE 13: (a) Average skin friction profiles for different values of $\lambda$ and Sc in transient state. (b) Average Nusselt number profiles for different values of $\lambda$ and Sc in transient state. (c) Average Sherwood number profiles for different values of $\lambda$ and Sc in transient state. 


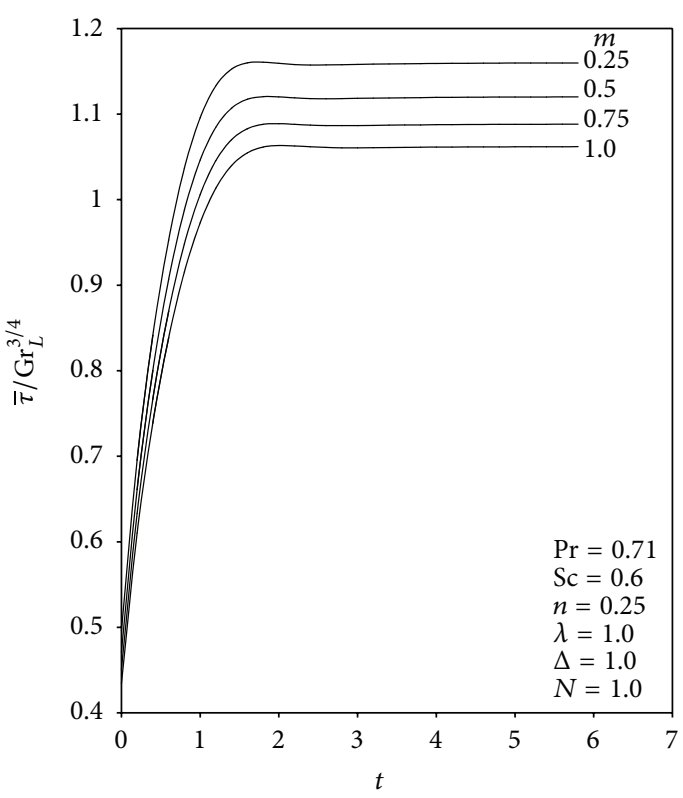

(a)

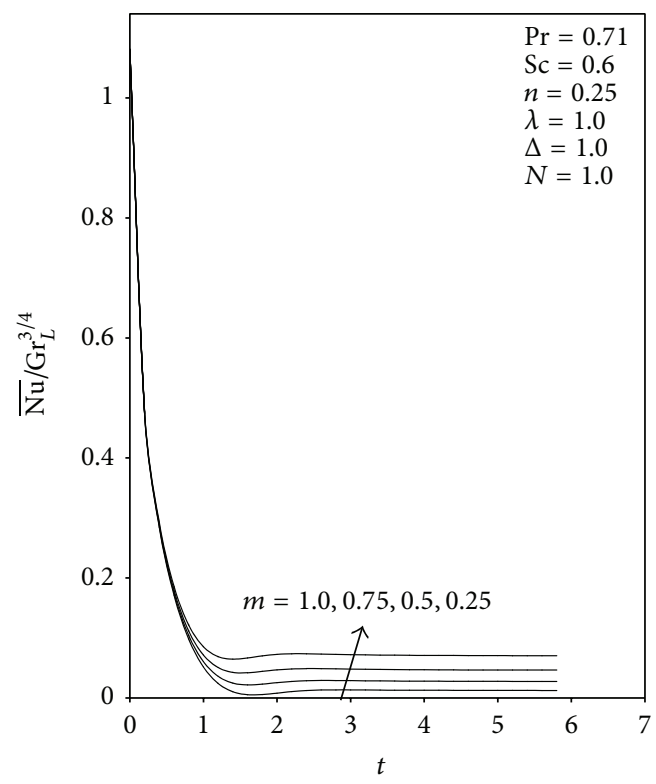

(b)

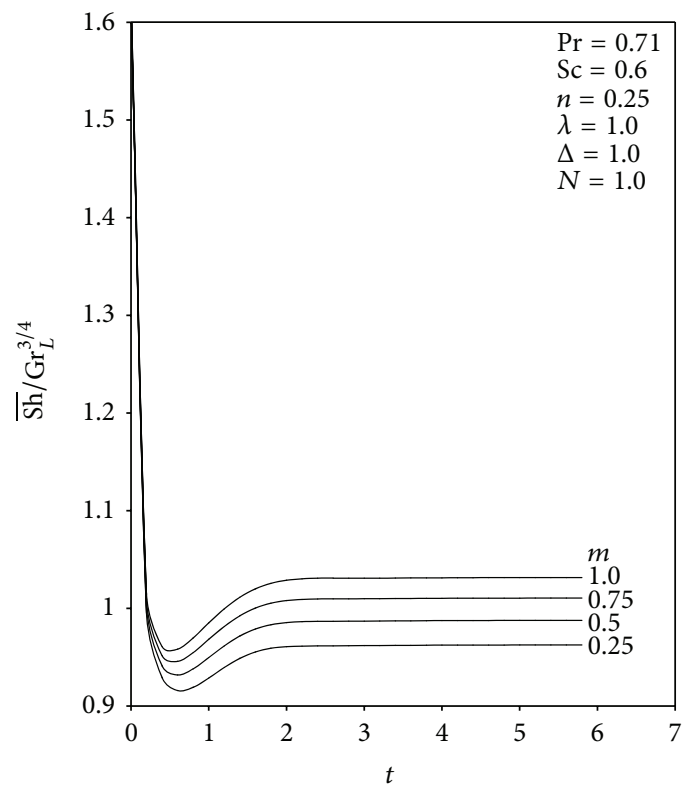

(c)

Figure 14: (a) Average skin friction profiles for different values of $m$ in transient state. (b) Average Nusselt number profiles for different values of $m$ in transient state. (c) Average Sherwood number profiles for different values of $m$ in transient state.

(Figure 7(b)), whereas the local Sherwood number increases for larger values of $\Delta$ and smaller values of $\operatorname{Pr}$ (Figure 7(c)). Figures $8(\mathrm{a})-8(\mathrm{c})$ depict the effects of chemical reaction parameter $\lambda$ and Schmidt number Sc on the local skin friction, local Nusselt number, and local Sherwood number. The local skin friction and local Nusselt number increase for smaller values of $\lambda$ and Sc. Increasing $\lambda$ and Sc clearly boosts the wall skin friction (Figure 8(a)). With increasing Sc, the local Nusselt number (Figure 8(b)) is consistently reduced. The surface species gradient, that is, mass transfer rate at the cone surface, is strongly elevated with a rise in $\lambda$ and $\mathrm{Sc}$ is observed from Figure 8(c). Figures 9(a)-9(c) show the effect of the surface concentration power law exponent $m$ on the local skin friction, local Nusselt number, and local Sherwood number. The local skin friction and local Nusselt number increase for smaller values of $m$, while the local Sherwood number increases for higher values of $m$. Figure 10 indicates the effect of surface temperature power law exponent $n$ on the local Sherwood number; it increases for larger values of $n$. It is observed from Figures 11(a)-11(c) the effect of buoyancy ratio parameter $N$ on the local skin friction, local Nusselt number, and local Sherwood number. Figure 11(a) illustrates a rise in 


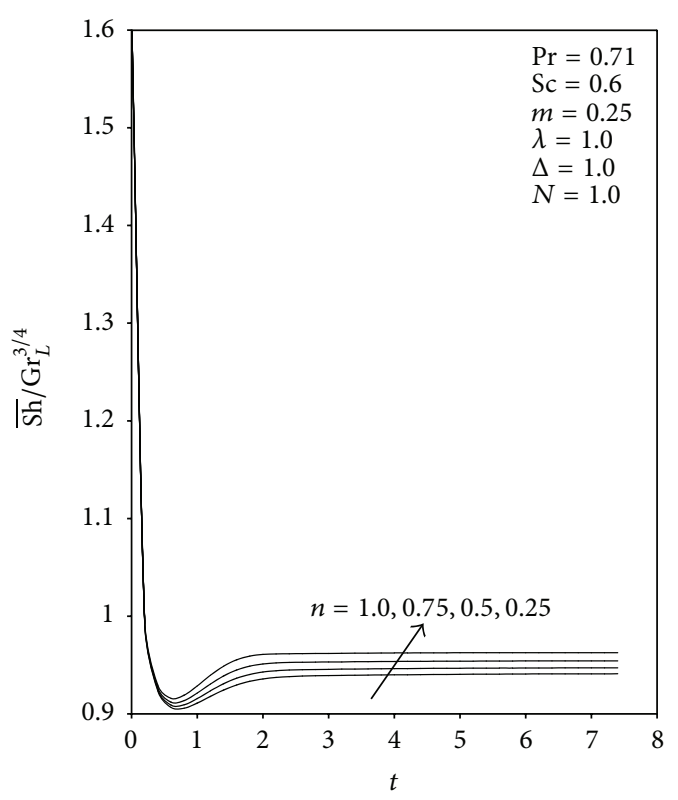

Figure 15: Average Sherwood number profiles for different values of $n$ in transient state.

$N$ accompanying a stronger increase in assisted buoyancy force; it strongly accelerates the flow and enhances the shear stress. The time required to attain the steady state is decreased with this increase in $N$. Inspection of Figures 11(b) and 11(c) shows that an increase in $N$ strongly boosts both $\mathrm{Nu}_{X}$ and $\mathrm{Sh}_{X}$; that is, it enhances the heat transfer gradient and mass transfer gradient at the cone surface. Further the Sherwood number increases with the increase in $N$. The physical reason is that positive force produced remarkable overshoot near the surface within the boundary layer for low Prandtl number fluid $(\mathrm{Pr}=0.71)$ but for high Prandtl number fluid $(\mathrm{Pr}=6.7)$ the velocity overshoot is not significant. Also, the buoyancy force enhanced the skin friction coefficient as well as the local Nusselt number or local heat transfer rate. Simultaneously the time required to attain the steady state is reduced with an increase in $N$ for both $\mathrm{Nu}_{X}$ and $\mathrm{Sh}_{X}$.

Time dependences of the average values of skin friction $\bar{\tau}$, Nusselt number $\overline{\mathrm{Nu}}$, and Sherwood number $\overline{\mathrm{Sh}}$ for various parameters $\operatorname{Pr}, \Delta, \lambda, \mathrm{Sc}, m, n$, and $N$ are plotted through Figures 12(a)-12(c) to Figures 16(a)-16(c). From Figures $12(a)-12(c)$, it is noticed that the effects of heat generation/absorption parameter $\Delta$ and $\operatorname{Pr}$ on the average skin friction. It increases for larger values of $\Delta$ and $\operatorname{Pr}$, whereas the average Nusselt number increases for smaller values of $\Delta$ and higher values of Pr. The average Sherwood number increases for larger values of $\Delta$ and smaller values of Pr. Figures 13(a)13(c) depict the effects of the chemical reaction parameter $\lambda$ and Schmidt number Sc on the average skin friction, average Nusselt number, and average Sherwood number. The average skin friction and Nusselt number increase for smaller values of $\lambda$ and Sc but the average Sherwood number increases for larger values of $\lambda$ and Sc. Figures 14(a)-14(c) show the effect of the surface concentration power law exponent $m$ on the average skin friction, average Nusselt number, and average
Sherwood number. The average skin friction and average Nusselt number increase for lower values of the surface concentration power law exponent $m$, but average Sherwood number increases for larger values of $m$. Figure 15 indicates the effect of surface temperature power law exponent $n$ on the average Sherwood number. The average Sherwood number increases for smaller values of $n$. It is clear from Figures 16(a)16(c) the average skin friction, average Nusselt number, and average Sherwood number increase for higher values of $N$.

\section{Conclusions}

A mathematical model has been presented for the free convection flow from a vertical cone with heat generation/absorption and chemical reaction. The family of governing partial differential equations is solved by an implicit finite difference scheme of Crank-Nicholson type. A parametric study is performed to illustrate the influence of thermophysical parameters on the velocity, temperature, and concentration profiles. It has been observed that

(1) the time taken to reach steady state increases with increasing $\Delta, \operatorname{Pr}, \lambda, S c, N, m$, and $n$;

(2) the fluid velocity increases for higher values of $\Delta, N$ and lower values of $\operatorname{Pr}, \lambda, \mathrm{Sc}$, and $m$. Temperature increases for larger values of $\Delta$, Sc and smaller values of $\operatorname{Pr}, \lambda, m$, and $N$, while the concentration of species decreases for smaller values of $\lambda, n$ and larger values of $\Delta, \operatorname{Pr}, \mathrm{Sc}, m$, and $N$;

(3) the local skin friction increases for higher values of $\operatorname{Pr}, \Delta$, and $N$ and for lower values of $\lambda$, Sc, and $m$. The local Nusselt number increases for higher values of $\mathrm{Pr}$ and $N$ and lower values of $\lambda$, Sc, $\Delta$, and $m$. The local Sherwood number increases for higher values of $\lambda, \mathrm{Sc}$, $\Delta, m, n$, and $N$ and smaller values of $\operatorname{Pr}$;

(4) the average skin friction increases for larger values of $\Delta, \operatorname{Pr}$, and $N$ and smaller values of $\lambda, \mathrm{Sc}$, and $m$. The average Nusselt number increases for higher values of Pr and $N$ and lower values of $\lambda$, Sc, $\Delta, m$, and $N$. The average Sherwood number increases for higher values of $\lambda, \mathrm{Sc}, \Delta, m$, and $N$ and lower values of $\operatorname{Pr}$ and $n$;

(5) momentum boundary layers become thick for higher values of Sc, $\Delta, \lambda$, and $N$ and lower values $\operatorname{Pr}$ and $m$, the thermal boundary layer becomes thick for higher values of Sc, $\Delta$, and $m$ and lower values of $\operatorname{Pr}, \lambda$, and $N$, and the concentration boundary layer becomes thick for larger values of $\lambda$ and $n$ and smaller values of $\Delta, \operatorname{Pr}, \mathrm{Sc}, N, m$, and $N$.

\section{Nomenclature}

$a: \quad$ Constant

b: $\quad$ Constant

$C_{P}$ : $\quad$ Specific heat at constant pressure

$D: \quad$ Mass diffusivity $\mathrm{m}^{2} \mathrm{~S}^{-1}$

$f^{\prime \prime}(\infty, 0)$ : Local skin friction in [8]

$\mathrm{Gr}_{L}$ : Thermal Grashof number 


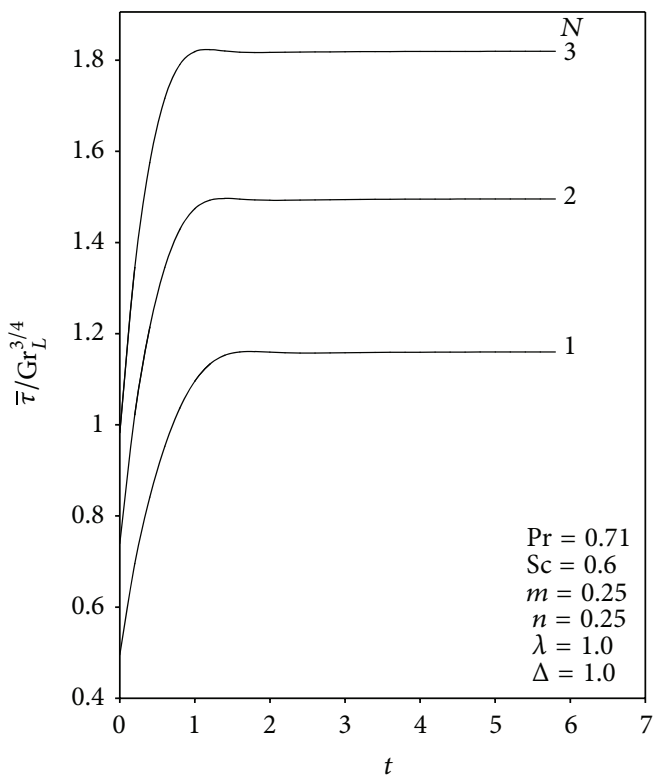

(a)

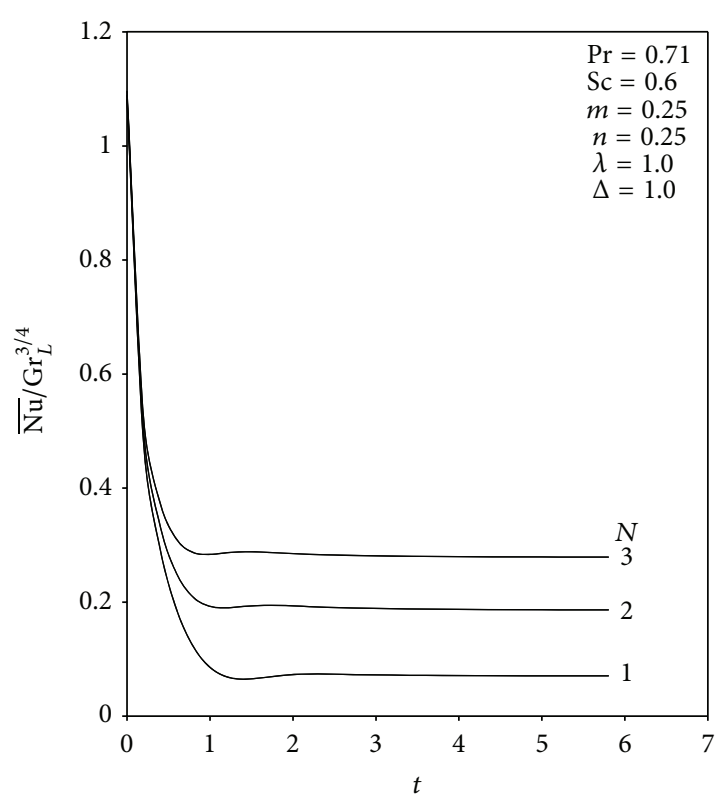

(b)

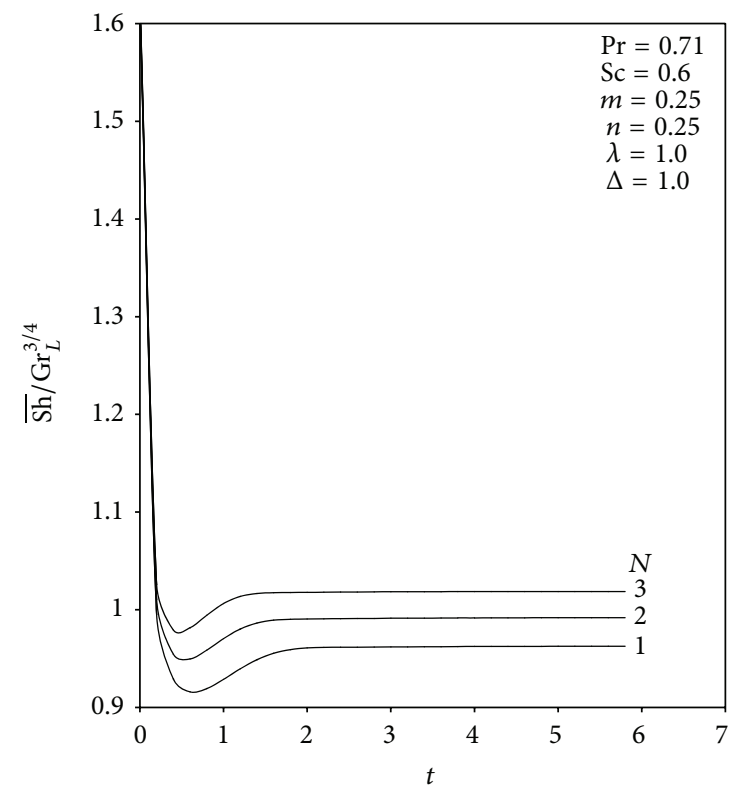

(c)

FIGURE 16: (a) Average skin friction profiles for different values of $N$ in transient state. (b) Average Nusselt number profiles for different values of $N$ in transient state. (c) Average Sherwood number profiles for different values of $N$ in transient state.

Gr*: Mass Grashof number

$g:$ Acceleration due to gravity $\mathrm{ms}^{-2}$

$k$ : Thermal conductivity $\mathrm{Wm}^{-1} \mathrm{~K}^{-1}$

$k_{1}$ : Dimensional chemical reaction parameter $J$

$L: \quad$ Reference length $m$

$m$ : Exponent in power law variation in surface concentration

$n$ : Exponent in power law variation in surface temperature

$N$ : Dimensionless buoyancy ratio
$\mathrm{Nu}_{x}$ : Local Nusselt number

$\overline{\mathrm{Nu}_{L}}$ : Average Nusselt number

$\mathrm{Nu}_{x}$ : Nondimensional local Nusselt number

$\overline{\mathrm{Nu}}$ : Nondimensional average Nusselt number

Pr: Prandtl number

$Q_{0}$ : Dimensional heat generation/absorption coefficient $\mathrm{Wm}^{-3}$

$R: \quad$ Dimensionless local radius

$r$ : Local radius of the cone $\mathrm{m}$ 
TABLE 1: Comparison of steady-state local skin friction and local Nusselt number values at $X=1.0$ with those of Chamkha [8] for full cone, for various values of $\operatorname{Pr}$ when $n=0, M=0, N=0$ and $R_{d}=0$.

\begin{tabular}{|c|c|c|c|c|}
\hline \multirow[b]{2}{*}{$\operatorname{Pr}$} & \multicolumn{2}{|c|}{ Local skin friction } & \multicolumn{2}{|c|}{ Local Nusselt number } \\
\hline & $\begin{array}{c}\text { Chamkha [8] } \\
f^{\prime \prime}(\infty, 0)\end{array}$ & $\begin{array}{c}\text { Present values } \\
\tau_{X} / \mathrm{Gr}_{L}^{3 / 4}\end{array}$ & $\begin{array}{c}\text { Chamkha [8] } \\
-\theta^{\prime}(\infty, 0)\end{array}$ & $\begin{array}{l}\text { Present results } \\
\mathrm{Nu}_{X} / \mathrm{Gr}_{L}{ }^{1 / 4}\end{array}$ \\
\hline 0.001 & 1.5135 & 1.4149 & 0.0245 & 0.0294 \\
\hline 0.01 & 1.3549 & 1.3356 & 0.0751 & 0.0797 \\
\hline 0.1 & 1.0962 & 1.0911 & 0.2116 & 0.2115 \\
\hline 1 & 0.7697 & 0.7688 & 0.5111 & 0.5125 \\
\hline 10 & 0.4877 & 0.4856 & 1.0342 & 1.0356 \\
\hline 100 & 0.2895 & 0.2879 & 1.9230 & 1.9316 \\
\hline 1000 & 0.1661 & 0.1637 & 3.4700 & 3.5186 \\
\hline
\end{tabular}

Sc: Schmidt number

$T^{\prime}$ : Temperature $\mathrm{K}^{0}$

$T$ : Dimensionless temperature

$t^{\prime}$ : Time s

$t$ : Dimensionless time

$U$ : Dimensionless velocity in $-X$ direction

$u$ : Velocity component in $x$ direction $\mathrm{ms}^{-1}$

$V$ : Dimensionless velocity in $-Y$ direction

$v$ : Velocity component in $-y$ direction $\mathrm{ms}^{-1}$

$X$ : Dimensionless spatial coordinate along the cone generator

$x$ : Spatial coordinate along the cone generator $\mathrm{m}$

$Y$ : Dimensionless spatial coordinate along the normal to the cone generator

$y$ : Spatial coordinate along the normal to the cone generator $\mathrm{m}$.

Greek Symbols

$\begin{array}{ll}\alpha: & \text { Thermal diffusivity } \mathrm{m}^{2} \mathrm{~s}^{-1} \\ \beta: & \text { Volumetric thermal expansion }{ }^{0} \mathrm{k}^{-1} \\ \beta^{*}: & \text { Volumetric coefficient of expansion with } \\ & \text { Concentration }{ }^{0} \mathrm{k}^{-1} \\ \Delta: & \quad \text { Dimensionless heat generation and } \\ & \text { absorption parameter } \\ \lambda: & \text { Nondimensional chemical reaction } \\ & \text { parameter } \\ \rho: & \text { Density kg } \mathrm{m}^{-3} \\ -\theta^{\prime}(\infty, 0): & \text { Local Nusselt number in }[8] \\ \Delta t: & \text { Dimensionless time step } \\ \Delta X: & \text { Dimensionless finite difference grid size in } \\ & X \text { direction } \\ \Delta Y: & \text { Dimensionless finite difference grid size in } \\ & \text { Y direction } \\ \mu: & \text { Dynamic viscosity } \mathrm{kg} \mathrm{m}^{-1} \mathrm{~s}^{-1} \\ \nu: & \text { Kinematic viscosity } \mathrm{m}^{2} \mathrm{~s}^{-1} \\ \tau_{x}: & \text { Local skin friction } \\ \tau_{X}: & \text { Dimensionless local skin friction }\end{array}$

$\overline{\tau_{L}}:$ Average skin friction

$\bar{\tau}$ : Dimensionless average skin friction.

Subscripts

$w$ : Condition on the wall

$\infty$ : Free stream condition.

\section{Conflict of Interests}

The authors declare that there is no conflict of interests regarding the publication of this paper.

\section{Acknowledgments}

The authors thankfully acknowledge the financial support from UTM-Flagship Research Grant, Vote no. 01G40, $\mathrm{MOHE}$, and Research Management Centre (RMC), Universiti Teknologi Malaysia for completion of this research work and thanks to the reviewers for their valuable suggestions and comments to improve this paper.

\section{References}

[1] H. J. Merk and J. A. Prins, "Thermal convection in laminary boundary layers I," Applied Scientific Research, vol. 4, pp. 11-24, 1953.

[2] H. J. Merk and J. A. Prins, "Thermal convection laminar boundary layer II," Applied Scientific Research, vol. 4, pp. 195206, 1954.

[3] R. G. Hering and R. J. Grosh, "Laminar free convection from a non-isothermal cone," International Journal of Heat and Mass Transfer, vol. 5, no. 11, pp. 1059-1068, 1962.

[4] R. G. Hering, "Laminar free convection from a non-isothermal cone at low Prandtl numbers," International Journal of Heat and Mass Transfer, vol. 8, no. 10, pp. 1333-1337, 1965.

[5] N. G. Kafoussias, "Effects of mass transfer on free convective flow past a vertical isothermal cone surface," International Journal of Engineering Science, vol. 30, no. 3, pp. 273-281, 1992.

[6] K. A. Yih, "Uniform transpiration effect on combined heat and mass transfer by natural convection over a cone in saturated 
porous media: Uniform wall temperature/concentration or heat/mass flux," International Journal of Heat and Mass Transfer, vol. 42, no. 18, pp. 3533-3537, 1999.

[7] K. A. Yih, "Coupled heat and mass transfer by free convection over a truncated cone in porous media: VWT/VWC or VHF/VMF," Acta Mechanica, vol. 137, no. 1, pp. 83-97, 1999.

[8] A. J. Chamkha, "Coupled heat and mass transfer by natural convection about a truncated cone in the presence of magnetic field and radiation effects," Numerical Heat Transfer A: Applications, vol. 39, no. 5, pp. 511-530, 2001.

[9] A. J. Chamkha and M. M. A. Quadri, "Combined heat and mass transfer by hydromagnetic natural convection over a cone embedded in a non-Darcian porous medium with heat generation/absorption effects," Heat and Mass Transfer/Waerme- und Stoffuebertragung, vol. 38, no. 6, pp. 487-495, 2002.

[10] A. A. Afify, "The effect of radiation on free convective flow and mass transfer past a vertical isothermal cone surface with chemical reaction in the presence of a transverse magnetic field," Canadian Journal of Physics, vol. 82, no. 6, pp. 447-458, 2004.

[11] A. J. Chamkha and A. Al-Mudhaf, "Unsteady heat and mass transfer from a rotating vertical cone with a magnetic field and heat generation or absorption effects," International Journal of Thermal Sciences, vol. 44, no. 3, pp. 267-276, 2005.

[12] A. J. Chamkha, A. F. Al-Mudhaf, and I. Pop, "Effect of heat generation or absorption on thermophoretic free convection boundary layer from a vertical flat plate embedded in a porous medium," International Communications in Heat and Mass Transfer, vol. 33, no. 9, pp. 1096-1102, 2006.

[13] S. M. M. El- Kabeir, M. Modather, and M. A. Mansour, "Effect of heat and mass transfer on free convection flow over a cone with uniform suction or injection in micro polar fluids," International Journal of Applied Mechanics and Enginering, vol. 11, no. 1, pp. 15-35, 2006.

[14] S. M. M. El-Kabeir and M. M. M. Abdou, "Chemical reaction, heat and mass transfer on MHD flow over a vertical isothermal cone surface in micropolar fluids with heat generation/absorption," Applied Mathematical Sciences: Journal for Theory and Applications, vol. 1, no. 33-36, pp. 1663-1674, 2007.

[15] S. M. M. El-Kabeir, M. A. El-Hakiem, and A. M. Rashad, "Group method analysis for the effect of radiation on MHD coupled heat and mass transfer natural convection flow water vapor over a vertical cone through porous medium," International Journal of Applied Mathematics and Mechanics, vol. 3, no. 2, pp. 35-53, 2007.

[16] C. Cheng, "Natural convection heat and mass transfer from a vertical truncated cone in a porous medium saturated with a non-Newtonian fluid with variable wall temperature and concentration," International Communications in Heat and Mass Transfer, vol. 36, no. 6, pp. 585-589, 2009.

[17] C. Y. Cheng, "Soret and Dufour effects on natural convection heat and mass transfer from a vertical cone in a porous medium," International Communications in Heat and Mass Transfer, vol. 36, no. 10, pp. 1020-1024, 2009.

[18] C. Cheng, "Soret and Dufour effects on heat and mass transfer by natural convection from a vertical truncated cone in a fluidsaturated porous medium with variable wall temperature and concentration," International Communications in Heat and Mass Transfer, vol. 37, no. 8, pp. 1031-1035, 2010.
[19] A. S. N. Murti, P. K. Kameswaran, and K. T. Poorna, "Radiation, chemical reaction, double dispersion effects on heat and mass transfer in non-Newtonian fluids," International Journal of Engineering, vol. 4, no. 1, pp. 13-25, 2010.

[20] P. M. Kishore, V. Rajesh, and S. Vijayakumar Verma, "Viscoelastic buoyancy- driven MHD free convective heat and mass transfer past avertical cone with thermal radiation and viscous dissipation effects," International Journal of Mathematics and Mechanics, vol. 6, no. 15, pp. 67-87, 2010.

[21] A. Mahdy, "Effect of chemical reaction and heat generation or absorption on double-diffusive convection from a vertical truncated cone in porous media with variable viscosity," International Communications in Heat and Mass Transfer, vol. 37, no. 5, pp. 548-554, 2010.

[22] S. G. Mohiddin, S. Vijayakumar Verma, and N. Ch. S. N. Iyengar, "Radiation and mass transfer effects on MHD free convective flow past a vertical cone with variable surface conditions in the presence of viscous dissipation," International Electronic Engineering Mathematical Society, vol. 8, pp. 22-37, 2010.

[23] S. Gouse Mohiddin, V. R. Prasad, S. V. K. Varma, and O. Anwar Bég, "Numerical study of unsteady free convective heat and mass transfer in a Walters- $B$ visco elastic flow along a vertical cone," International Journal of Applied Mathematics and Mechanics, vol. 6, no. 15, pp. 88-114, 2010.

[24] P. M. Patil and I. Pop, "Effects of surface mass transfer on unsteady mixed convection flow over a vertical cone with chemical reaction," International Journal of Heat Mass Transfer, vol. 47, no. 11, pp. 1453-1464, 2011.

[25] S. M. M. EL-Kabeir and E. A. EL-Sayed, "Effects of thermal radiation and viscous dissipation on MHD viscoelastic free convection past a vertical isothermal cone surface with chemical reaction," International Journal of Energy \& Technology, vol. 4, no. 10, pp. 1-7, 2012.

[26] F. G. Awad, P. Sibanda, S. S. Motsa, and O. D. Makinde, "Convection from an inverted cone in a porous medium with cross-diffusion effects," Computers \& Mathematics with Applications, vol. 61, no. 5, pp. 1431-1441, 2011.

[27] M. Narayana, F. G. Awad, and P. Sibanda, "Free magnetohydrodynamic flow and convection from a vertical spinning cone with cross-diffusion effects," Applied Mathematical Modelling. Simulation and Computation for Engineering and Environmental Systems, vol. 37, no. 5, pp. 2662-2678, 2013.

[28] A. Basiri Parsa, M. M. Rashidi, and T. Hayat, "MHD boundarylayer flow over a stretching surface with internal heat generation or absorption," Heat Transfer-Asian Research, vol. 42, no. 6, pp. 500-514, 2013.

[29] V. M. Soundalgekar and P. Ganesan, "Finite-difference analysis of transient free convection with mass transfer on an isothermal vertical flat plate," International Journal of Engineering Science, vol. 19, no. 6, pp. 757-770, 1981.

[30] B. Pullepu, K. Ekambavanan, and A. J. Chamkha, "Unsteady laminar natural convection from a non-isothermal vertical cone," Nonlinear Analysis: Modelling and Control, vol. 12, no. 4, pp. 525-540, 2007.

[31] R. Muthukumaraswamy and P. Ganesan, "Unsteady flow past an impulsively started vertical plate with heat and mass transfer," International Journal of Heat Mass Transfer, vol. 34, no. 2-3, pp. 187-193, 1998. 
[32] R. Muthukumaraswamy and P. Ganesan, "Natural convection on a moving isothermal vertical plate with chemical reaction," Journal of Engineering Physics and Thermophysics, vol. 75, no. 1, pp. 113-119, 2002.

[33] P. Bapuji, K. Ekambavanan, and I. Pop, "Finite difference analysis of laminar free convection flow past a non isothermal vertical cone," Heat and Mass Transfer, vol. 44, no. 5, pp. 517$526,2008$. 


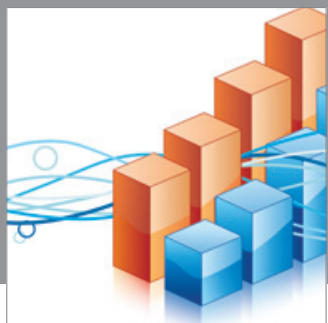

Advances in

Operations Research

mansans

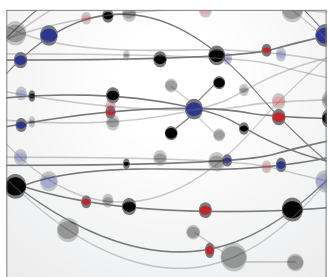

The Scientific World Journal
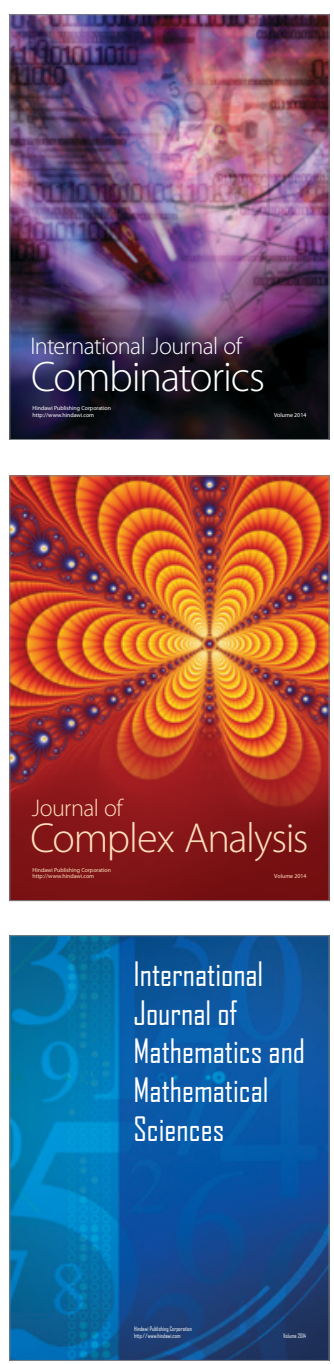
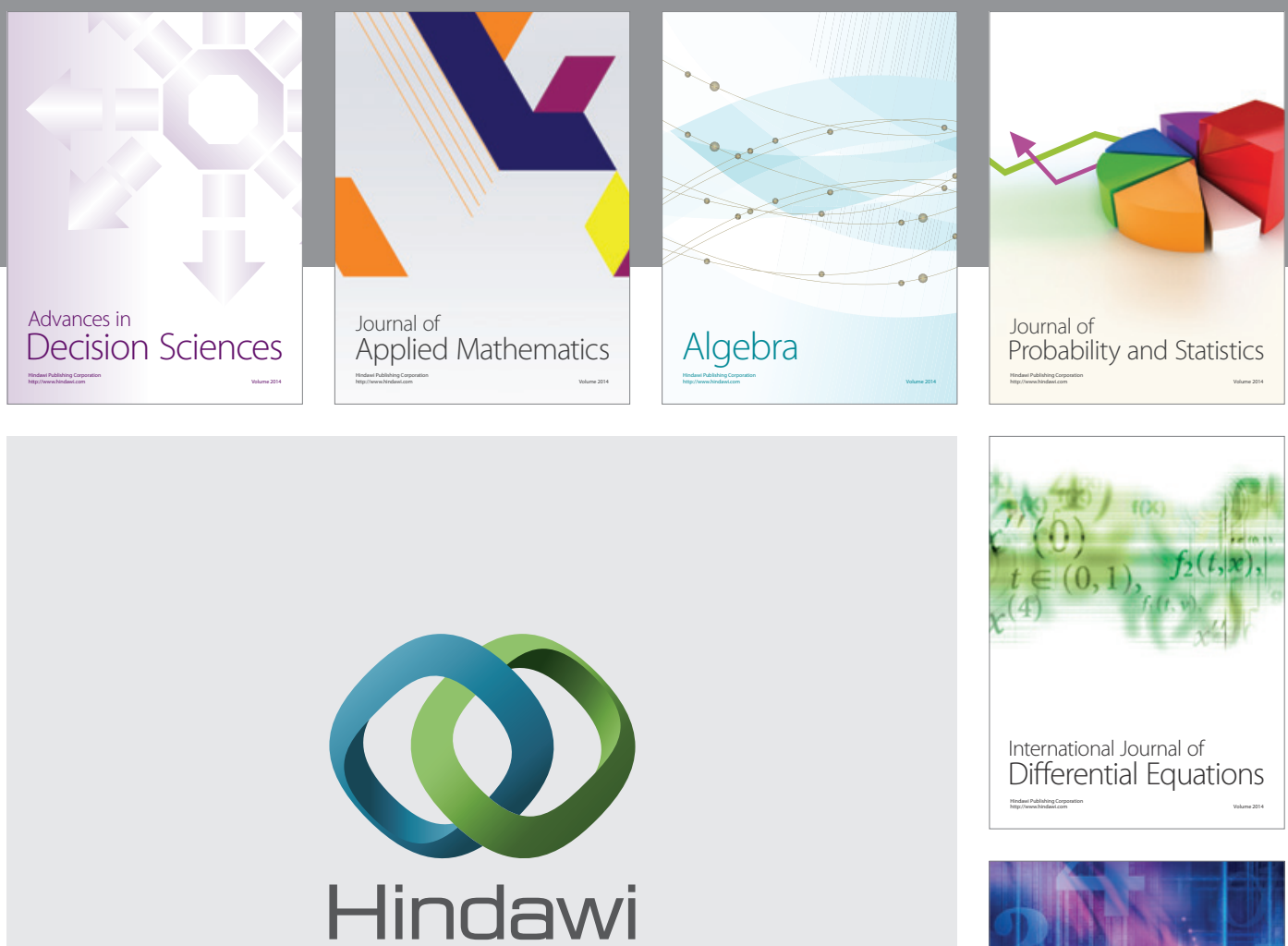

Submit your manuscripts at http://www.hindawi.com
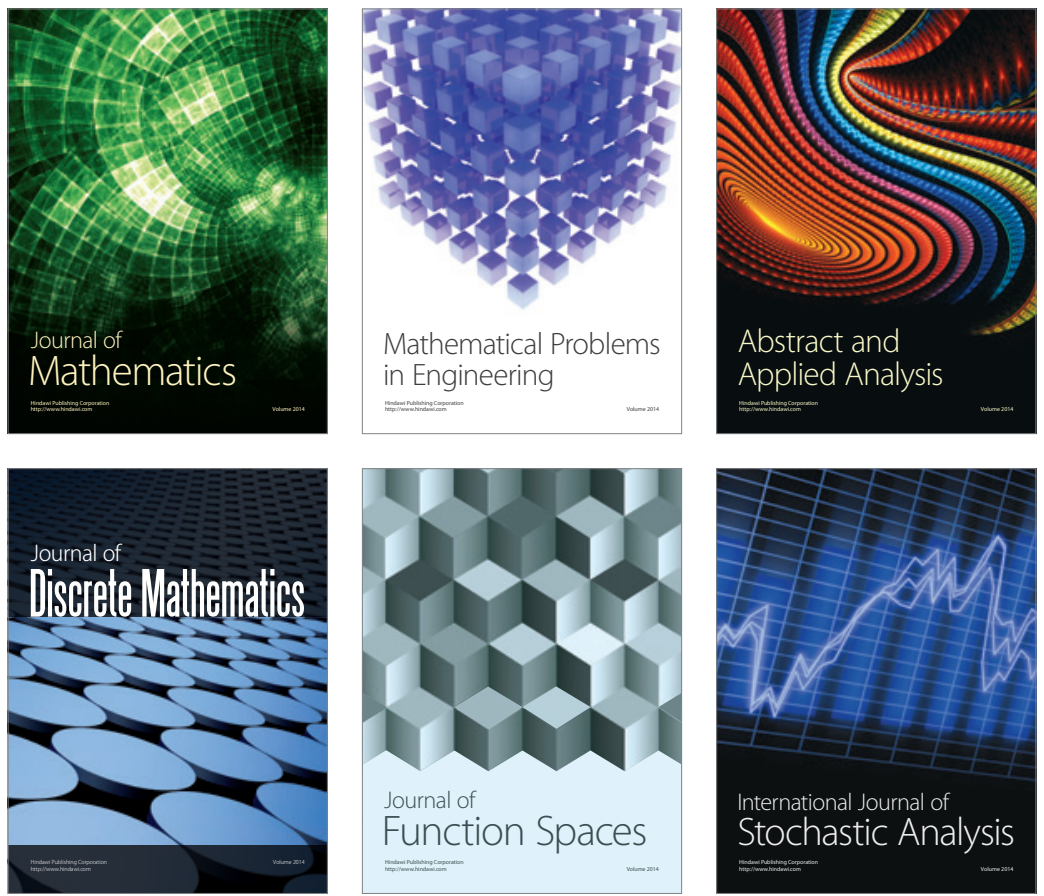

Journal of

Function Spaces

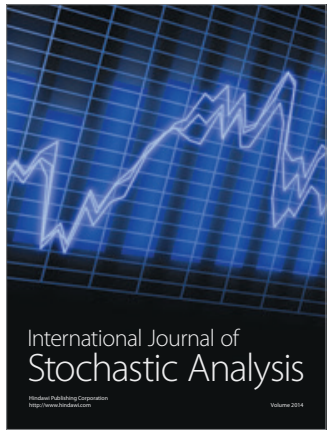

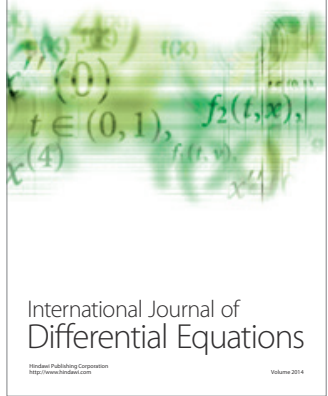
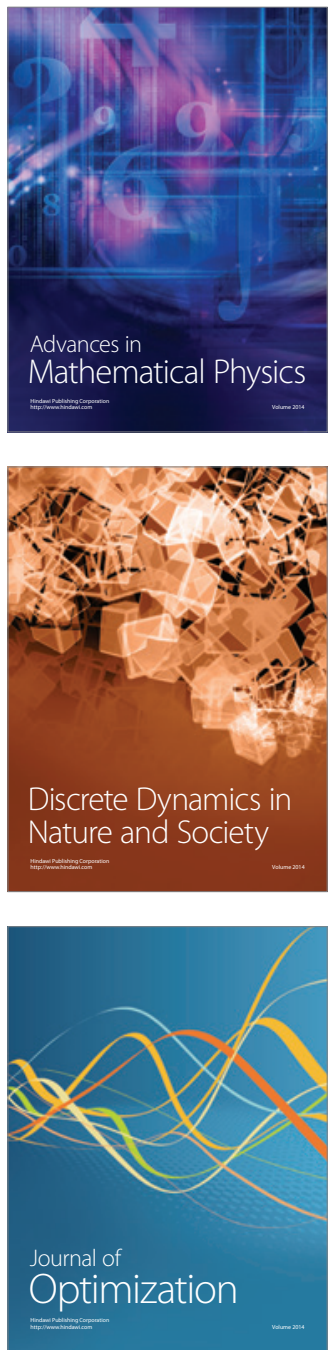